\title{
Dian Schefold zur (direkten) Demokratie
}

Zugleich Besprechung von: Dian Schefold: Bewahrung der Demokratie. Ausgewählte Aufsätze, hrsg. v. Nikolaos K. Hlepas/Paolo Ridola/Alfred Rinken/Gerhard Stuby/Luzius Wildhaber, Berliner Wissenschafts-Verlag: Berlin 2012, 502 S., 74 Euro.

\section{Otmar Jung}

\section{Einleitung}

Dian Schefold ist überzeugter Schweizerbürger - nicht weil seine Vorfahren seit Generationen im Alpenraum gelebt hätten, sondern weil seine Eltern die Eidgenossenschaft als freies Land erlebten, als im damaligen Deutschen Reich Diskriminierung und Verfolgung herrschten ${ }^{1}$. Schweizerisch an Schefold ist gewiß sein Engagement für direkte Demokratie, von dem schon seine Dissertation in Basel 1961 zeugt $^{2}$. Seit Jahrzehnten lebt und lehrt Schefold in Deutschland, 1980 bis 2001 als Professor für Öffentliches Recht, einschließlich allgemeine Staatslehre und neuere Verfassungsgeschichte, mit dem Schwerpunkt Verwaltungsrecht am Fachbereich Rechtswissenschaft der Universität Bremen ${ }^{3}$, der Stadt, die ihm ,zur neuen Heimat“ wurde ${ }^{4}$. Nimmt man zur frühen schweizerischen Prägung und den langen Berufsjahren in Deutschland noch sein Arbeitsgebiet der deutsch-italienischen Rechtsvergleichung hinzu, erklärt sich eine ganz spezifische Haltung, die Schefold einnimmt: Zu seinem stupenden Wissen kommen ein scharfer Blick und eine Nüchternheit des Urteils, die mit seiner Distanz zu tun haben, die offenbar manches besser erkennen läßt. Der gleiche Abstand zeigt

Vgl. Karl Schefold, Die Dichtung als Führerin zur klassischen Kunst. Erinnerungen eines Archäologen, hrsg. v. Martha Rohde-Liegle in Verbindung mit Dian, Reimar und Bertram Schefold, 2003 (Lebenserinnerungen Bd. 58), S. 75-85. In der Zeitgeschichtswissenschaft war die causa in den Grundzügen bekannt seit Reinhard Bollmus, Das Amt Rosenberg und seine Gegner. Studien zum Machtkampf im nationalsozialistischen Herrschaftssystem, 1970 [2. Aufl. 2006] (Studien zur Zeitgeschichte Bd. 1), S. 171, 307 Fn. 61a.

2 Vgl. Dian Schefold, Volkssouveränität und repräsentative Demokratie in der schweizerischen Regeneration 1830-1848, 1966 (Basler Studien zur Rechtswissenschaft H. 76).

3 Siehe auch die Skizzen von Lebenslauf und wissenschaftlichem Wirken im Vorwort der Herausgeber der Festschrift: Demokratie und Selbstverwaltung in Europa. Festschrift für Dian Schefold zum 65. Geburtstag, hrsg. von Andreas Bovenschulte/Henning Grub/Franziska Alice Löhr/Matthias v. Schwanenflügel/Wiebke Wietschel, 2001, S. 7-10. A.a.O., S. 8. 
sich auch in der hier vornehmlich interessierenden Perspektive: Einerseits kann man Schefold ohne Übertreibung als Mentor in Sachen direkte Demokratie in Deutschland bezeichnen ${ }^{5}$. Andererseits war er immer vorsichtig, was eine Übertragbarkeit der schweizerischen Direktdemokratie angeht ${ }^{6}$, und hat er eine spezifische Zurückhaltung beobachtet, wenn es etwa um Mitgliedschaften ${ }^{7}$ oder allzu deutliche Parteinahme in Deutschland ging. $\mathrm{Zu}$ den verfassungs- und rechtspolitischen Bestrebungen im Zusammenhang mit der deutschen Einheit, direktdemokratische Einwirkungsformen durchzusetzen, konstatierte er beispielsweise rückblickend: „Als - wenn auch in Deutschland lehrender - Schweizer hatte ich insofern Bedenken, mich an dieser politischen Debatte zu beteiligen, aus allgemeinen Erwägungen wie auch in Kenntnis der doch recht grundlegenden Strukturunterschiede ... ich kann und muß mich als Außenstehender betrachten“ (S. 377).

Diese Vorgeschichte ist wichtig, um das hier zu besprechende Buch richtig zu erfassen. Es heißt nicht „Bewahrung der direkten Demokratie“, aber die „,ausgewählten Aufsätze“ lassen das Engagement des Verfassers immer wieder erkennen. Selbstredend stellt es nur einen Teil aus Schefolds wissenschaftlichem Euvre dar ${ }^{8}$, und es sind auch keineswegs alle Arbeiten zur direkten Demokratie versammelt ${ }^{9}$.

5 Exemplarisch mag hier die Situation bei den zwei Fachgesprächen über „Direkte Demokratie" im wiedervereinigten Deutschland erscheinen, die 1990 in der Evangelischen Akademie Hofgeismar stattfanden. In dem respektablen Kreis - die Teilnehmer (darunter der Rezensent) sind aufgeführt bei: Direkte Demokratie in Deutschland. Handreichungen zur Verfassungsdiskussion in Bund und Ländern. Mit Entwürfen zur Einführung von Volksbegehren und Volksentscheid auf Bundesebene („Hofgeismarer Entwurf“), hrsg. von der Evangelischen Akademie Hofgeismar/Stiftung Mitarbeit, 1991 (BrennpunktDokumentation Nr. 12), S. 89 - war Schefold zwar nicht der einzige Hochschullehrer, wohl aber der einzige Staatsrechtsprofessor.

6 Vgl. seine nachdenkliche Betrachtung: Exportartikel Referendumsdemokratie? Schweizer Wissenschaftler in Deutschland als Vertreter, in: Peter Rück (Hrsg.), Grenzerfahrungen. Schweizer Wissenschaftler, Journalisten und Künstler in Deutschland, 1991, S. 167-177. Wie anders dagegen der Ton eines acht Jahre später in der Schweiz erarbeiteten Bandes: Gebhard Kirchgässner/Lars P. Feld/Marcel R. Savioz, Die direkte Demokratie: Modern, erfolgreich, entwicklungs- und exportfähig, 1999.

7 Beispielsweise auch um die (ihm angetragene, aber von ihm abgelehnte) Mitgliedschaft im Kuratorium für Mehr Demokratie.

8 Näher dazu die Vorbemerkung des Verfassers (S. 11), vor allem zum Ausschluß aller monographischen und fremdsprachlichen Veröffentlichungen. Für Schefolds Schriftenverzeichnis bis Anfang 2001 sei verwiesen auf: Demokratie und Selbstverwaltung in Europa. Festschrift, 2001, S. 445-463. Aktualisiert findet es sich unter http://www.jura.unibremen./tdeypo3/cms405/index.php?id=217 (Zugriff 20.3.2012).

9 Beispielsweise fehlen sein Beitrag: Mehr direkte Demokratie wagen? Verfassungsrechtliche Probleme plebiszitärer Entscheidungsverfahren im parlamentarischen System, in: Hans-Dieter Klingemann/Wolfgang Luthardt (Hrsg.), Wohlfahrtsstaat, Sozialstruktur und Verfassungsanalyse, 1993, S. 189-205, erweitert veröffentlicht u.d.T.: Politische Mitwirkung der Bürger auf Landesebene, in: ZParl 20 (1989) (!), S. 425-441, der seinerzeit viel 


\section{Geschichte der Staatstheorien und Verfassungen ${ }^{10}$}

Den ganzen Band leitet ein der Beitrag über „Rousseaus doppelte Staatslehre“, zuerst erschienen in der Gedenkschrift für Max Imboden 1972. In eingehender Auseinandersetzung mit der Literatur und immer eng an Rousseaus Texten argumentierend - am gesamten Werk: über die klassischen Schriften hinaus von den Entwürfen bis zu den posthum veröffentlichten Schriften und zu den Briefen, und das mit geradezu philologischer Akribie -, arbeitet Schefold die beiden Zweige von Rousseaus Staatslehre heraus. Sie beschäftigt sich einerseits mit der kleinen, am antiken Staatsideal ausgerichteten freien „Republik“, die bestimmte soziale und geographische Voraussetzungen verlangt, und andererseits mit dem monarchisch-absolutistischen Großstaat seiner Gegenwart. Dahinter scheinen die Stadtrepublik Genf seiner Jugend auf und das Königreich Frankreich, in dem er die aktivste Erwachsenenzeit verbrachte. Schefold betont, daß Rousseau sich im praktischen Ergebnis „weitgehend als Anhänger der bestehenden politischen Ordnungen, als Konservativer" zeige (S. 25), wenngleich dieser Konservativismus entgegengesetzte Funktionen habe: „In der Republik gilt es, Sorge zu tragen, daß alle Veränderungen der Institutionen und der Lebensweise die Homogenität des Volkes und die einende Kraft des Staates nicht schwächen ... Die Bewahrung des Bestehenden dient hier der Identifikation von Staat und Bürger sowie der Festigung der Herrschaft des Gesetzes. Umgekehrt sichert im absolutistischen Großstaat die Bewahrung des Bestehenden die Ruhe und damit den Freiraum, in dem das Individuum sich entfalten kann, ohne mit dem Staat in Konflikt zu geraten.“(S. 26).

$\mathrm{Da}$ diese doppelte Staatslehre mißverstanden werden konnte, wundert nicht. So schrieb sich etwa - pointiert Schefold - ,der dem Fortschritt zugewandte Flächenstaat die Aufgabe zu, die Staatstheorie zu verwirklichen, die Rousseau für den traditionsgebundenen Kleinstaat entwickelt hatte. Augenfälliges Beispiel eines solchen Rollenwechsels war die Berufung auf Rousseau in der französischen Revolution ..., aber auch die Durchsetzung des repräsentativ-demokratischen Bundesstaats in der Schweiz gegen den Widerstand unmittelbar demokratischer Kantone.“(S. 29).

Ist das alles nur noch Geschichte der Staatslehre? So fragt Schefold abschließend, „ob denn jene sozialen Voraussetzungen auch heute die einzig denkbaren für die Errichtung einer Republik sind und ob nicht die Veränderungen der Ver-

beachtet wurde; seine erwähnte Betrachtung „Exportartikel Referendumsdemokratie?“ (1991) oder die neue Darstellung: Die Neuregelung des Volksentscheids in Bremen vom 1. September 2009, in: JdD 2 (2010), S. 135-154. - Manches mag er dabei als zu speziell empfunden haben.

10 Die Zwischenüberschriften entsprechen den Kapitelbezeichnungen des besprochenen Bandes. 
hältnisse seit der Mitte des 18. Jahrhunderts erlauben, die Grundgedanken der Republik auf den modernen Flächenstaat zu übertragen“" (S. 31). Dazu gibt er zwei ermutigende Antworten: „Im Bereich der staatlichen Organisation und Willensbildung hat die schweizerische Referendumsdemokratie eine solche Übertragung längst vollzogen. Ihre Institutionen ermöglichen den kontinuierlichen Austausch der Meinungen zur Bildung eines Staatswillens, der den Anspruch erheben kann, sich einer volonté générale anzunähern, die Entpolitisierung des Bürgers zu erschweren.“ (S. 31). Und bei den sozialen Voraussetzungen gibt Schefold zu erwägen, „ob nicht die Leistungen des Staates ..., vor allem auf dem Gebiet des Erziehungswesens und des wirtschaftlichen Ausgleichs, einen politischen Gemeinschaftssinn erhalten und erneuern können, der die alte Ambiance des Contrat social ersetzen, die im Discours sur l'inégalité aufgezeigte Entwicklung abwenden kann“" (S. 31 f.).

Eine würdige Gabe des 36 Jahre jungen Wissenschaftlers und Hochschullehrers für seinen vor der Zeit verstorbenen Doktorvater!

Der folgende Aufsatz „,Von der Relativität des Staatsbegriffs. Ein Blick zurück auf Rousseau - und nach vorn “, mehr als drei Jahrzehnte später geschrieben und ursprünglich Gerhard Stuby gewidmet, dem bremischen Kollegen, sucht die damalige Argumentation fortzuführen, und zwar exemplarisch an der volonté générale. Im Kleinstaat, dem Modell der Republik des Contrat social, werden die besonderen Willen fast nur negativ bewertet; Ziel ist, sie zugunsten des Gemeinwillens zu brechen. Aber gegenüber den Großstaaten seiner Zeit betrachtet Rousseau auch einen Gemeinwillen kleinerer Gruppen - er nennt Klerus, Militär und Adel, aber könnte man nicht auch an die Kommunen denken? - und die überstaatliche Ordnung, „einen Gemeinwillen im Weltmaßstab“ (S. 39), als „nicht weniger legitim“ (S. 41). Diese ,parallele Legitimation des Gemeinwillens auf verschiedenen Ebenen“ - ,innerstaatlicher Gruppierungen, des Staates und der Weltgemeinschaft“ - ermöglicht, so Schefold, „eine neue, eigenständige Begründung des Völkerrechts“ (S. 40). Rousseau könne so „als Staatsdenker gedeutet werden, der - bei voller Anerkennung der Bedeutung des Souveränitätsprinzips - angesichts der Wirklichkeit des modernen, die Ungleichheit nicht überwindenden Staates diesem das Völkerrecht als eigenständig legitimierte Rechtsordnung der Weltgemeinschaft entgegenstellt" (S. 44).

Dieses Kapitel beschließt der Aufsatz „,Verfassung als Kompromiß? Deutung und Bedeutung des preußischen Verfassungskonflikts “, zuerst 1981 in der „Zeitschrift für Neuere Rechtsgeschichte“ erschienen. Eingangs entfaltet Schefold die Kontroverse zwischen Ernst Rudolf Huber, der die deutsche konstitutionelle Monarchie als einen tragfähigen, dauerhaften Kompromiß der Verfassungsordnung deutete, und einer vor allem von Ernst-Wolfgang Böckenförde begründeten 
Antithese, wonach der den preußischen Verfassungskonflikt beendende Kompromiß von 1866 nur „brüchig, transitorisch“ gewesen sei (S. 49). Diese Antithese wird eingehend dargelegt und mit weiteren Indizien gestärkt, aber Schefold führt auch gute Argumente für die Stabilität an. In einer Zwischenbilanz neigt er der Kritik an der Tragfähigkeit des konstitutionell monarchischen Staates zu, mit zwei Einschränkungen: Quantitativ gefragt, also nach der Leistung und Stabilität, sollte man „die Bedeutung der dem Kaiserreich zugrunde liegenden Verfassungsstrukturen nicht gering“ achten (S. 58). Qualitativ müsse man fragen, „ob nach dem preußischen Verfassungskonflikt die durch das Indemnitätsgesetz wiederhergestellte Einheit wirksam, wirklich genug war, um die Verfassungen Preußens und des Reichs zur Grundlage einer funktionsfähigen, legitimen politischen Ordnung zu machen“" (S. 59). Hier bemerkt der Jurist - wie Schefold in dieser zu einem guten Teil von Neuhistorikern und Sozialgeschichtlern bestrittenen Debatte betont - ein „Zurückbleiben der Leistung hinter dem Anspruch“, eine „Spannung zwischen Verfassungswirklichkeit und Verfassungsrecht" (S. 59).

Zum Konflikt selbst arbeitet Schefold Unstimmigkeiten im Verhältnis zur Verfassungsurkunde heraus, er argumentiert überzeugend gegen Bismarcks, von Huber akzeptierte „Lückentheorie“ und stellt heraus, daß für die Entscheidung eines Konflikts der Kammern mit der Regierung das konstitutionelle Staatsrecht eigentlich „Verfahren richterlicher Entscheidung in Reserve“ hielt (S. 66). Daß dies in Preußen nicht bestand bzw. ihre Einführung von Bismarck ausdrücklich abgelehnt wurde, belegt für Schefold, daß die konstitutionelle Monarchie eben „kein einheitliches, dauerndes Baugesetz“ hatte (S. 67). Der schließlich gefundene Kompromiß besaß zwar eine „faktische Konsistenz“; entscheidend ist für Schefold allerdings die Frage, ob er darüber hinaus ,auch als verfassungsrechtliche Ordnung den Anspruch darauf erheben konnte, die Kräfte der Nation auf Dauer zu integrieren“ (S. 68). Diese Frage läßt sich allerdings nun klar verneinen. 1866 kam es eben zu keinem „Verfassungskompromiß“, der die aufgebrochenen Verfassungsfragen geklärt hätte, sondern man sah nur „die taktische Beilegung einer Kontroverse zwischen Regierung und Abgeordnetenhaus“ auf der haushaltsrechtlichen Ebene (S. 71). Für diese Situation erscheint Schefold der Begriff des „Scheinkonstitutionalismus“ zutreffend, den er dahingehend präzisiert, daß „das positive Verfassungsrecht die monarchische Kommandogewalt und damit die soziopolitische Funktion des Heeres, [die] mögliche Überspielung des parlamentarischen Budgetrechts und die Sanktion von Verfassungsverletzungen der Exekutive nicht hinreichend regelte“ (S. 69). Die Konsequenzen waren enorm. Mit dem Heer, ,einem von ,blindem Gehorsam“ geprägten Instrument ..., blieb der gewichtigste Teil des staatlichen Machtpotentials der Verfassungsordnung entzogen“ (S. 69). Bedenkt man die Wirkung dieses „Vorbilds“ für die Beamtenschaft und den Adel, ergab sich: „In der geschriebenen Verfassung nicht voll erfaßte Kräfte, die dennoch für das konstitutionelle System unab- 
dingbar waren, hingen mit ihren Prärogativen und existentiellen Vorbehalten wie ein Damoklesschwert über der ordentlichen, der normativen Verfassungsordnung.“ Es trat, wie Schefold bewußt ,in Vorwegnahme späterer schlimmerer Formen“ formuliert, „eine zweite Art der Staatlichkeit neben den Normenstaat". Der Verfassungsstaat wurde insofern transitorisch, als er „dem Zugriff eines Trägers der Gewalt des Ausnahmezustandes ausgeliefert“ war (S. 70). Die „Kardinalfrage“, „ob der Verfassungskompromiß auch tatsächlich die politisch und wirtschaftlich relevanten Kräfte der Gesellschaft einbezieht, normiert und ihnen ihren Ort zuweist" (S. 71), beantwortet sich damit von selbst negativ.

Schefold zeigt sich mit diesem Beitrag nicht nur bestens beschlagen in der preußisch-deutschen Verfassungsgeschichte; man kann auch in der Art seiner Fragen und seiner Antworten einen wohltuenden Einfluß schweizerischen Abstands spüren.

\section{Demokratietheorie}

Dieses Kapitel beginnt mit einem Text, den Schefold 1974 zunächst in Basel als Vortrag hielt und im selben Jahr in der „Zeitschrift für Schweizerisches Recht“ veröffentlichte: ,Bewahrung der Demokratie und wirtschaftlich-technische Entwicklung“. Schefold fragt dabei ,nach einem normativen Demokratiebegriff, nach einer effektiveren Kontrolle der wirtschaftlich-technischen Entwicklung“ (S. 81). Im historischen Zugriff arbeitet er kundig die wirtschaftliche wie die demokratische Entwicklung vor allem in Preußen/Deutschland und in der Schweiz heraus. Insbesondere der schweizerischen Sozialgeschichte wie der politischen Ideengeschichte des 19. Jahrhunderts lasse sich, so Schefold, „ein normativer Demokratiebegriff entnehmen“ (S. 83). Im folgenden werden die Wandlungen des Rechtsstaats und der Funktion der Grundrechte, der Verwaltung wie innerhalb der Schweiz der Volksrechte eingehend behandelt; Schefold erörtert die Möglichkeiten, „die Fragestellung und Entscheidungsfindung im politischen Prozeß, in Regierung, Parlament und Volk zu verbessern“ (S. 92), und empfiehlt die Demokratisierung der gesellschaftlichen Großorganisationen analog dem einschlägigen Verfassungsgebot für die Parteien (Art. 21 GG). Den Abschluß bildet eine gute Abwägung der seinerzeit geplanten Ausweitung der betrieblichen Mitbestimmung in Deutschland.

Auch dies ist wieder ein gewichtiger Beitrag, der mit Recht für den Band ausgewählt wurde, und doch merkt man ihm, anders als den geistes- bzw. politikgeschichtlichen Aufsätzen des 1. Kapitels, deutlich an, daß 38 Jahre seitdem vergangen sind. Einiges mag heute hellsichtig erscheinen, etwa sein Wort vom ,fait accompli der administrativ-technischen Entscheidungsfindung“ (S. 89), und „daß der Willensbildungsproze $\beta$ in Parlament und Volk erst einsetze, wenn die Ent- 
scheidungen gefallen sind“ (S. 80). Manches dagegen wirkt überholt. Die Idee des „Junktims zwischen Demokratisierung und wirtschaftlich-technischer Entwicklung“ (S. 85) mochte vor vier Jahrzehnten unmittelbar einleuchten; heute blickt man nach China und fragt sich, ob hinter ihr nicht eine (west-)europäische Engführung steckte. Daß Schefold für eine „Bereitschaft zur Demokratie als Staats- und Gesellschaftsform des Ausgleichs, zur Bescheidung und Selbstbeschränkung“ (S. 99) plädierte und die „Bereitschaft, Sachzwänge in Frage stellen zu lassen“ (S. 101), als Ausweis demokratischer Staatsgesinnung pries, kommt einem geradezu utopisch vor. Der Autor hatte eben noch die klassische Industrialisierung vor Augen, sprach mit Respekt von der Montanindustrie - unvorstellbar, daß heute eine globalisierte Finanzindustrie die Regierungen Europas vor sich her treibt, die zur Begründung ihrer immer neuen Projekte zur „Rettung“ der Gemeinschaftswährung Euro den Volksvertretungen nicht mehr zu sagen wissen, als daß dies ,alternativlos“ sei, und damit, wie ernsthaft zu befürchten ist, das Ende der parlamentarischen Demokratie herbeiführen ${ }^{11}$.

Apropos: Für die seinerzeitige, ungleich weniger scharfe Konfliktlage in der Schweiz stellte Schefold fest, daß ,sich die Ausdehnung der unmittelbaren Demokratie als Remedur untauglich erwiesen“ (S. 95) habe. Sollte das nicht den Hoffnungen auf Problemlösung, die mancher mit der Einführung der direkten Demokratie in Deutschland auf Bundesebene verbindet, einen Dämpfer versetzen?

Biographisch ist noch bemerkenswert, mit welcher honetten Begründung Schefold 1974 - im eingeschlossenen West-Berlin schreibend - die utopische Demokratietheorie marxistisch-leninistischer Prägung ablehnte: „weil sie für eine absehbare Übergangsperiode die Demokratie zurückstellen, der technischwirtschaftlichen Entwicklung den Vorrang einräumen muß, ohne daß sie absehbare Gewißheit über das Ende dieser Periode geben kann“ (S. 95).

Der Beitrag ,,Volk als Tatsache, Ideologie und politische Kultur“ geht auf einen Vortrag zurück, den Schefold 1995 bei der Konferenz „Volk - Nation - Europa. Zur Romantisierung und Entromantisierung politischer Begriffe" in Amsterdam gehalten hat (hier nach dem Tagungsband 1998). Als Jurist vor einem primär literaturwissenschaftlichen Forum nahm er sich drei Fragen vor: „1.) wer wie über die Zusammensetzung des Volkes bestimmt, 2.) wie diese Bestimmung andere Zugehörigkeiten verdrängen kann und 3.) ob sie dies tun muß“ (S. 102). Die Antworten sind gediegen: Staatsgewalt und Volk bedingen einander. „Wenn jene vom Volk ausgehen soll, muß sie doch bestimmen, wer zum Volk gehört" (S. 103). Das Erfahrungsmaterial zu diesem Problemkreis ist freilich weithin nega-

11 Vgl. Herfried Münkler, Die rasenden Politiker. Vom absehbaren Ende der parlamentarischen Demokratie, in: Der Spiegel Nr. 29 v. 16.7.2012, S. 100 f. 
tiv, sei es, daß ethnische Besonderheiten im Namen der Nation verschwiegen und damit unterdrückt wurden (klassisches Beispiel: die „Bretonen, Basken und Elsässer in Frankreich“, S. 104), sei es, daß die gerade zur Vortragszeit virulente Ideologie der ,ethnischen Reinheit“ bis zu den Verbrechen entsprechender „Säuberungen“ im Gebiet des ehemaligen Jugoslawiens trieb (S. 106).

Schefold wendet sich engagiert gegen den „Ausschließlichkeitscharakter" des Volksbegriffs, der vor allem auf Abgrenzung aus ist (S. 108). Von daher kann er auch von einem „Gemeindevolk“ wie von ,einem europäischen Volk“ sprechen (S. 106) und die Mehrstaatlichkeit bejahen, wobei er gegen Befürchtungen, hier könnten „Rechte kumuliert werden“, für eine Lösung wie im Internationalen Privatrecht plädiert: „eine Prävalenz des Wohnsitzstaats“ (S. 107). Unverkennbar, daß diese Optionen auch eigene existentielle Erfahrungen des Autors widerspiegeln. Abschließend plädiert Schefold für ,die Aufgabe geistiger - künstlerischer und wissenschaftlicher - Führung und Prägung des Volkes“ (S. 102). Volk sei „,insofern kein abgeschlossener, ein für allemal feststehender Befund, sondern er konstituiert und konkretisiert sich in täglicher Praxis“" (S. 108).

Acht Jahre später sprach Schefold bei dem wiederum von der „Stiftung für Romantikforschung“ veranstalteten Kolloquium über „Ungleichzeitigkeiten der Europäischen Romantik“ auf Naxos (2003) zum Thema: „Konstitutionelle Monarchie als Staatsform der Romantik?" (hier nach dem Tagungsband 2006). Wie in dieser Überschrift zu fragen, erscheint Schefold legitim. Die immerhin hundert Jahre (von der Deutschen Bundesakte 1815 bis zur Novemberrevolution 1918) bestehende konstitutionelle Monarchie wurde in der Tat - als positiv gedeuteter deutscher Sonderweg („Überlegenheit deutscher Staatskunst“, S. 114) - „,nachträglich romantisierend verklärt" (S. 126). Aber das konstitutionell-monarchische Verfassungskonzept ist nicht aus romantischem Denken entstanden, sondern folgte wesentlich aufklärerischen Gedanken (S. 117). Gerade umgekehrt wäre für jenes ein Problem, wie sich eine auf das monarchische Prinzip gegründete Legitimität „mit konstitutioneller Bindung und Beschränkung der Fürsten vertrug“ (S. 120). Schefold nennt dafür realpolitische Gründe, vor allem die fortwirkenden Folgen napoleonischer Umwälzungen, hält aber auch eine ,romantische Haltung“ (S. 121) für möglich - insofern vermag er, wie er freimütig einräumt, keine „eindeutige Antwort zu geben“ (S. 109). Die abschließend aufgeworfene Frage, ob die konstitutionelle Monarchie ein eigenständiger Verfassungstyp gewesen sei, bejaht Schefold, auf seine ausführlichere Darstellung von 1981 verweisend, im Grundsatz, wiederholt freilich den Vorwurf des „Scheinkonstitutionalismus“" (S. 126). 
Dieses Kapitel schließt mit dem (ganz neuen ${ }^{12}$ ) Beitrag „Die Homogenität im Mehrebenensystem. Kontinuität und Kohärenz der argumentativen Entwicklung unter besonderer Berücksichtigung von Hugo Preuß" (1860-1925). Schefold holt hier weit aus, um darzutun, daß die Souveränitätslehre zu einer „Sonderstellung des Staates gegenüber allen übrigen Ebenen“ führt; er ist eben „Träger eines Gewaltmonopols“ (S. 129). Dies bedeutet, daß sowohl nach unten - vor allem die kommunale Ebene - wie nach oben - das Völkerrecht - eine Homogenität ausgeschlossen ist. Gleichwohl kann Schefold verfassungsgeschichtlich ein Bedürfnis nach Homogenität zeigen und greift zur theoretischen Fundierung auf Hugo Preuß und Hans Kelsen zurück.

Gewiß geht es dem Autor dabei vor allem, wie seine Überschrift deutlich macht, um die Fruchtbarmachung der Preußschen Lehren ${ }^{13}$; dennoch seien hier die praktischen Folgerungen hervorgehoben, die Schefold gerade in der Perspektive direkter Demokratie zieht: Zunächst konstatiert er mit schweizerischer Distanz, daß ,jedenfalls nach der in Deutschland vorherrschenden Meinung“ die Einführung direktdemokratischer Elemente für die kleineren Organisationseinheiten näher liege als für den Gesamtstaat (S. 142). Exakt dies ist ja der gegenwärtige Befund auf kommunalverfassungs- und landesverfassungsrechtlicher Ebene einerseits und auf Bundesebene andererseits (wobei man sich dann noch fragen mag, ob die Verfassungspolitik der vorherrschenden Meinung gefolgt ist oder ob die letztere in alter obrigkeitsstaatlicher Manier alles, was ist, will sagen: den jeweils erreichten Stand der politischen Auseinandersetzungen, verklärt).

Alsdann bemerkt er zu der geläufigen Kritik am Demokratiedefizit der Europäischen Union, daß in manchen „Mitgliedstaaten die demokratische Beteiligung nicht besser gewährleistet ist als auf europäischer Ebene“ (S. 148), wobei man mit Blick auf die Europäische Bürgerinitiative pointieren könnte, daß solche Partizipation in Deutschland auf Bundesebene noch nicht einmal so schwach eröffnet sei wie neuerdings in der EU.

12 Dieser - gewichtige - Text erschien im Jahrbuch des öffentlichen Rechts der Gegenwart Bd. 60 (2012). Ihn gleichzeitig als ausgewählten Aufsatz in den Band „Bewahrung der Demokratie“ aufzunehmen folgt offensichtlich einer anderen Überlegung als dem im Vorwort genannten Ansatz, der fachwissenschaftlichen Diskussion wertvolle, ,verstreute und teilweise nur schwer greifbare“ Aufsätze Schefolds aus den Jahren 1972 bis 2011 ,zugänglich“ zu machen.

13 Schefold hat seit zwei Jahrzehnten über Preuß geforscht und publiziert, angefangen von dem nachfolgend zu würdigenden Beitrag (1993) über den Höhepunkt des Bandes 2 von Preuß“ „Gesammelten Schriften“: „Öffentliches Recht und Rechtsphilosophie im Kaiserreich“, den Schefold 2009 herausgegeben und mit einer gewichtigen Einleitung (76 S.) versehen hat, bis zu dem gerade behandelten Beitrag (2012). - In seiner „Vorbemerkung“ hielt Schefold fest, daß ihn für die Thematik des hier besprochenen Bandes, ,vor allem die Auseinandersetzung mit Jean-Jacques Rousseau, Hugo Preuß und Hermann Heller beeinflußt" habe (S. 11). 
Interessant ist Schefolds abschließende Einschätzung des Vorschlags, den Mehr Demokratie e.V. unterbreitet hat ${ }^{14}$, Vertragsänderungen einer konsultativen Volksbefragung in der Europäischen Union insgesamt zu unterbreiten. Dazu entwirft er folgende Szenarien: Durch eine derartige Volksbefragung ,würden das förmliche Änderungsverfahren und die Entscheidungszuständigkeiten nicht verändert, wohl aber würde die demokratische Legitimation der Änderung auf europäischer Ebene bestärkt und präzisiert. Im Fall eines negativen Ausgangs läge nahe, das Änderungsverfahren durch Verzicht auf die Ratifikationen abzubrechen oder zumindest, entsprechend wie in Art. 48 V EUV vorgesehen, zu überdenken. Umgekehrt könnte ein positiver Ausgang den Mitgliedstaaten Anlass geben, auf einen nationalen Volksentscheid zu verzichten - oder diesen beeinflussen." (S. 149).

\section{Weimarer Staatsrechtslehre und ihre Bedeutung}

Zunächst steht hier der Beitrag „Hugo Preuß (1860-1925). Von der Stadtverfassung zur Staatsverfassung der Weimarer Republik", die erste Veröffentlichung Schefolds zu Preuß, erschienen in dem Sammelband „Deutsche Juristen jüdischer Herkunft“ (1993). Schefold baut auf dem bis dahin erreichten Stand der biographischen Forschung auf und bereichert das Bild durch die spezifischen Beobachtungen des Staatsrechtslehrers und Verfassungsgeschichtlers. Zum Werk des „Verfassungsvaters“ Preuß hält er fest, daß die Nationalversammlung von dem ersten Preußschen Entwurf in einigen Hinsichten abgewichen sei, u. a. insofern, als „das Verfassungsreferendum ... gestrichen [wurde], dafür weitere Formen der Volksgesetzgebung vorgesehen“ wurden; er bemerkt aber auch, daß „,von einer Ablehnung der Volksgesetzgebung durch Preuß ... dennoch, entgegen manchen Äußerungen in der Literatur, schwerlich gesprochen werden“ könne (S. 172). Die erstere Feststellung ist richtig, bleibt aber an der Oberfläche. Im selben Jahr 1993 zeigte der Rezensent, daß Preuß in der Volksabstimmung über eine bestimmte einzelne Frage ein „ermäßigende[s] Moment“ im Sinne einer Chance zur Versachlichung im Vergleich zu Wahlen erkannt ${ }^{15}$ und betont hatte, es liege ihm ,durchaus fern, etwa prinzipielle Gegengründe gegen den Gedanken des Referendums anzuführen“, und daß sein geflügeltes Wort vom „Damokles-

14 Vgl. Daniela Beer/Roman Huber, Wege zur Demokratisierung der Europäischen Union, in: Hermann K. Heußner/Otmar Jung (Hrsg.), Mehr direkte Demokratie wagen. Volksentscheid und Bürgerentscheid: Geschichte - Praxis - Vorschläge, 2. Auflage, 2009, S. 181-194 (187).

15 Otmar Jung, Die „Weimarer Erfahrungen“ mit der Volksgesetzgebung: Kritik und Tragweite. Stellungnahme zum Beitrag Meineke im Jahrbuch für Politik 1992, in: JfP 3 (1993), Halbbd. 1, S. 63-92 (73). 
schwert der reinen Demokratie“, das man nicht über das parlamentarische System hängen solle, sich nur auf die damals beantragte plebiszitäre Parlamentsauflösung bezog - was regelmäßig übersehen wird ${ }^{16}$.

An diesem Detail zeigt sich das Problem, das entstehen kann, wenn eine grundsätzlich sehr gute - Darstellung fast zwei Jahrzehnte später unverändert wieder gedruckt wird ${ }^{17}$, ja letztlich ein Problem des Konzepts „Ausgewählte Aufsätze“ überhaupt: Sie belegen sozusagen biographisch die wissenschaftliche Leistung des Autors - und das ist ja auch der primäre Zweck -, sie liefern Mosaiksteine zur Geschichte seines Faches, aber sie sind nicht - und können gar nicht sein - der letzte Stand der Wissenschaft.

Der folgende Beitrag „Geisteswissenschaften und Staatsrechtslehre zwischen Weimar und Bonn “, zuerst 1998 erschienen, gibt einen gelehrten Überblick über ein Dreivierteljahrhundert Fachgeschichte, wobei die Zeiten zwischen „Weimar“ und „Bonn“, nämlich die Zeit des Nationalsozialismus und die unmittelbare Nachkriegszeit, kritisch einbezogen werden. Hochschullehrer, Werke und Methoden, die Rechtsprechung des Bundesverfassungsgerichts, die in diesem Kontext erst verständlich wird, und schließlich der Ausblick insbesondere auf die Politikwissenschaft fügen sich zu einem beeindruckenden Bild, das viel Großes, aber auch nicht wenig allzu Menschliches enthält und die Einsicht, daß es unglaubliche Gemengelagen gibt: Für Gerhard Leibholz, wegen seiner jüdischen Herkunft gefährdet, als Kriegsteilnehmer privilegiert, schließlich doch seiner Professur enthoben, Schwager Dietrich Bonhoeffers, blieb „der italienische Faschismus noch mehrere Jahre eine diskussionswürdige Alternative“ (S. 187) ${ }^{18}$; 1938 Exil in England, 1947 Rückkehr an „seine“ Universität Göttingen, 1951 Richter am Bundesverfassungsgericht für 20 Jahre.

Es folgen noch zwei Beiträge über Hermann Heller (1892-1933), der ebenso wie Hugo Preuß für Schefold „eine gelungene Verbindung von emphatischem Verfassungspatriotismus und realistischem Blick für die sozial-ökonomischen Voraussetzungen und Grenzen der demokratischen Ordnung“ verkörpert ${ }^{19}$. In „, Hel-

16 A.a.O., S. 78 Fn. 3; vertieft bei Otmar Jung, Zur Revision der „Weimarer Erfahrungen“ mit der Volksgesetzgebung. Stellungnahme zum Beitrag Meineke im Jahrbuch für Politik 1994, in: JfP 5 (1995), Halbbd. 1, S. 67-116 (102 ff., 106).

17 Siehe zu diesem Problem allgemein Schefold in seiner ,Vorbemerkung“, S. 11 f. (12).

18 Eine bemerkenswerte Verbindung besteht hier dank der bei Schefold entstandenen Dissertation von Susanne Benöhr: Das faschistische Verfassungsrecht Italiens aus der Sicht von Gerhard Leibholz. Zu den Ursprüngen der Parteienstaatslehre, 1999 (Schriften zum Parteienrecht Bd. 23).

19 Vgl. das Vorwort zu: Demokratie und Selbstverwaltung in Europa. Festschrift, 2001, S. 10 . 
lers Ringen um den Verfassungsbegriff" (zuerst 1984 in der Gedächtnisschrift für Heller) zeichnet Schefold dessen Verfassungslehre anhand des letzten Kapitels seiner „Staatslehre“ nach, verortet sie mit ihrem sozialwissenschaftlichen Ansatz und ihrer innovativen Methodik im akademischen Umfeld der Weimarer Zeit - in der Art und souveränen Stoffbeherrschung wie im vorhergehenden Beitrag - und pointiert vor allem, daß sie in gewisser Weise die Antwort auf Carl Schmitts „Verfassungslehre“ von 1928 sei. Bei der Frage nach der Bedeutung von Hellers Verfassungsbegriff und den Folgerungen für die Gegenwart nimmt Schefold die „Staatslehre“, niedergeschrieben in der dramatischen Krise nach 1930, gegen den Vorwurf in Schutz, daß Heller hier „die Normativität prekär“ gemacht und womöglich „den Machtverhältnissen zu viel Spielraum gelassen“ habe. Vor dem düsteren zeithistorischen Hintergrund könne man Hellers Haltung „nicht resignativ, sondern allenfalls realistisch, wenn nicht verzweifelt optimistisch" nennen (S. 217).

Abschließend mustert Schefold noch Hellers Schüler sowie sein politisches Umfeld und bringt dabei manch Überraschendes: So zeigt er, daß Wolfgang Abendroth, der nach der sozialwissenschaftlichen Methode und seinen politischen Präferenzen Heller durchaus nahesteht, dessen Begriff des „sozialen Rechtsstaats" methodisch in der Manier Carl Schmitts einsetzt (S. 223).

Der Beitrag „,Gesellschaftliche und staatliche Demokratietheorie. Bemerkungen zu Hermann Heller " erschien 16 Jahre später. Schefold arbeitet wieder theoriegeschichtlich beeindruckend, nach seinen Ergebnissen könnte man allerdings zunächst die Hauptüberschrift fast für ironisch halten: „Das Konzept gesellschaftlicher Demokratie“ spiele bei Heller „,nur eine marginale Rolle“ (S. 232), und auch für die staatliche Demokratietheorie kommt Schefold zu einem ,magere(n) Befund" (S. 241). Dieser theoriegeschichtlichen Enttäuschung stehen freilich die Leistungen Hellers bei „konkreten verfassungsrechtlichen Fragen“ gegenüber; „in der praktischen staatsrechtlichen Argumentation [hat er] als Demokrat dazu beigetragen ..., die Verfassungsstrukturen der Weimarer Demokratie zu stärken“ (S. 241 f.). Zu würdigen blieben staatstheoretisch immerhin Hellers Begriff der Repräsentation, des Gesetzes und des sozialen Rechtsstaats. Vor allem sah Heller - wie Schefold rühmt -, daß Deutschland vor der „Entscheidung zwischen faschistischer Diktatur und sozialem Rechtsstaat“ stand: „Die Erkenntnis dieser Alternative 1929/30 gehört zu den Wegmarken deutscher Geschichte.“ (S. 243 f.).

Abschließend zeigt Schefold aber auch bei den Leistungen noch Schattenseiten. Daß Heller „die verselbständigte Staatsgewalt, die Organisation als Wirkungseinheit, die Repräsentation“ betonte, während die „Legitimation des gesellschaftlichen Wirkungszusammenhangs zum Staat zurückgedrängt wurde“, erlaubte gravierende Folgerungen: „Wenn ,repräsentative Demokratie als eigentli- 
che Form der Demokratie" verstanden wird, so kann sich diese These durchaus auf Hermann Heller berufen.“ (S. 244) - womit wir bei Böckenförde ${ }^{20}$ und einigen Landesverfassungsgerichten angelangt wären ${ }^{21}$.

\section{Verfassungsrecht der Bundesrepublik Deutschland}

Der einleitende Beitrag „Normenkontrolle und politisches Recht“ von 1972 ist zusammen mit dem ersten Rousseau-Text der früheste der hier versammelten Aufsätze. Zunächst schildert Schefold die Entwicklung der Verfassungsgerichtsbarkeit zur echten Rechtsprechung: beginnend mit den Vorgaben der Weimarer Reichsverfassung, verstärkt durch das vom Reichsgericht usurpierte Recht der Normenkontrolle Mitte der 1920er Jahre, fortgeführt durch die Entscheidung des Parlamentarischen Rats 1948/49 für ein Bundesverfassungsgericht und abschlieBend mit der Stellung, die dieses Gericht in den ersten 20 Jahren seiner Judikatur errang. Einher damit ging schon in der Weimarer Zeit ein „Wandel der Verfassungsinterpretation, vor allem des Grundrechtsverständnisses“. Daß Richter nun „sozialgestaltende Normen auf [eine] Verletzung des Gleichheitssatzes und der Garantie des Privateigentums" prüften (S. 254), hatte freilich eine erhebliche Brisanz: Schefold spricht deutlich von dem ,durch Standes- und Klasseninteressen motivierten Versuch [...] der Richter, politische Entscheidungen an sich zu ziehen, an Stelle des dazu demokratisch besser legitimierten parlamentarischen Gesetzgebers“ (S. 260) ${ }^{22}$. Nach 1945 waren wichtige Rahmenbedingungen anders, aber auch nun lassen sich viele Auseinandersetzungen um die Grundrechtsbindung des Gesetzgebers nur verstehen, wenn man hinter den dogmatischen und methodologischen Kontroversen die konkreten Interessen(-gruppen) erkennt, de-

20 Vgl. Ernst-Wolfgang Böckenförde: Mittelbare/repräsentative Demokratie als eigentliche Form der Demokratie. Bemerkungen zu Begriff und Verwirklichungsproblemen der Demokratie als Staats- und Regierungsform, in: Georg Müller/René A. Rhinow/Georg Schmid/Luzius Wildhaber (Hrsg.), Staatsorganisation und Staatsfunktionen im Wandel. Festschrift für Kurt Eichenberger zum 60. Geburtstag, 1982, S. 301-328.

21 Ein jüngstes Beispiel für die Aktualität Hellers bietet - angesichts der Finanzkrise - Albrecht Dehnhard, Das Versagen der Staaten und die Staatslehre Hermann Hellers, in: RuP 46 (2010), S. 179-182.

22 Als Exempel nennt er allerdings nur die Aufwertungsklagen nach der großen Inflation (S. 249 f.). Die unselige Wirkung der geradezu skandalösen Fürstenprozesse, die dann bis zur „sozialen Explosion“ des Volksentscheids zur Fürstenenteignung 1926 führten, war von der Juristischen Zeitgeschichte noch nicht aufgearbeitet, vgl. Otmar Jung, Volksgesetzgebung. Die „Weimarer Erfahrungen“ aus dem Fall der Vermögensauseinandersetzungen zwischen Freistaaten und ehemaligen Fürsten, 2. Aufl. 1996 (Studien zur Geschichtsforschung der Neuzeit Bd. 1), S. 585-625. 
nen diese oder jene Interpretation nützen sollte, wie Schefold am Beispiel all derer zeigt, die nun eine „Einrichtungsgarantie“ beanspruchten (vgl. S. 255 f.).

Abschließend fragt Schefold, nachdem er die historische Verifikation und die Beschränkung auf die klassischen Auslegungsregeln des Positivismus geprüft und verworfen hat, wie es möglich sein könne, „Auslegungsergebnisse allgemein überzeugend herzuleiten und methodisch zu fundieren“" (S. 265). Als Antwort plädiert er für eine Methodenkombination, bei der erst durch juristische Verfassungsauslegung das Normprogramm geklärt (,welche Werte und Interessen die Verfassung hier zur Bewertung und Feststellung aufgibt") und dann mit sozialwissenschaftlichen Methoden der Normbereich erhellt wird (,die tatsächlichen Interessen feststellen und ihren Gehalt bewerten“). Danach sei „eine Subsumtion möglich, die die Chance eröffnet, Vorverständnisse des Juristen zu überwinden und plausibel zu sein“ (S. 266).

Der folgende Beitrag „Der Bundesrat als konkordanzpolitisches Stabilisierungsinstrument ", zuerst 1992 erschienen, stellt zunächst Entstehung und Funktion des Bundesrats in der Entwicklung deutscher Bundesstaatlichkeit dar, beginnend mit dem Reichstag des Heiligen Römischen Reichs Deutscher Nation, durchgehalten in der Weimarer Reichsverfassung, die das Prinzip abschwächte, aber nicht demokratisierte, und aus nachvollziehbaren Gründen vom Parlamentarischen Rat wiederaufgenommen, freilich um einen gewissen Preis: „Der deutsche Föderalismus ist mit der Hypothek der lokalen Souveräne belastet" (S. 280). Danach behandelt Schefold die Zusammensetzung, Organisation und Verfahrensweise des Bundesrats, führt seine Zuständigkeiten auf und erklärt ihre Bedeutung, und schließt mit der Erörterung der Funktion und des politischen Gewichts des Bundesrats im Verfassungssystem Deutschlands. Dies alles ist gediegen dargestellt, alle Problempunkte werden angesteuert: der Bundesrat als „Instrument der Selbstkoordination der Länderverwaltungen“ (S. 274), die gewaltige Ausdehnung der Zahl der zustimmungspflichtigen Gesetze durch die Rechtsprechung des Bundesverfassungsgerichts zu Art. 84 Abs. 1 GG, „die paradoxe Lage, daß die Ausweitung der Bundesgesetzgebung den Föderalismus in der Form der Beteiligung des Bundesrats verstärkt hat" (S. 278), und der Wandel der Tätigkeit des Bundesrats von der Mitsprache zur Entwicklung einer „Alternative zur Regierungspolitik“ (S. 281), ja der Wandel der Funktion dieses ganzen Bundesorgans vom Schutz typischer Länderinteressen zur Funktion „eines zusätzlichen alternativen Machtzentrums“ (S. 282).

Man könnte es bei einem Lob dieses guten Bundesrats-Beitrags belassen, wäre da nicht ein Begriff: Ist der Bundesrat wirklich gemäß der Überschrift ein „konkordanzpolitisches“ Instrument? Der Terminus taucht nur noch einmal am Schluß des Textes auf in der folgenden Argumentation: Noch über den Erfolg bei Bundestagswahlen hinaus werde „der Nachweis der Regierungsfähigkeit in 
den Ländern zum entscheidenden Legitimationskriterium für politische Parteien. Dies zwingt alle Parteien zur Mäßigung, zur Homogenisierung ihrer Politik und zur Kooperationsbereitschaft. Dadurch entsteht ein Machtkartell, das Stabilität und Kontinuität gewährleistet. Es schließt Alternativen nicht aus, aber es hält das Ausmaß und die Tragweite der Alternativität in den engen Schranken der Konkordanzdemokratie.“ (S. 283). Wenn ein Schweizer Wissenschaftler so schreibt, stutzt man. Die schweizerische Konkordanzdemokratie ist - grob skizziert - gekennzeichnet durch einen geringen Parteienwettbewerb (außerhalb von Wahlen), Parlamentswahlen haben keinen Einfluß auf die Zusammensetzung der Regierung, es gibt keinen Machtwechsel zwischen Regierung und Opposition, und es herrscht das Prinzip der proportionalen Machtteilung. All dies traf und trifft für Deutschland nicht zu. Ferner ist das System der Konkordanzdemokratie in der Schweiz entstanden als Reaktion der politischen Elite auf den intensiven Gebrauch der „Volksrechte“ - diese gibt es im schweizerischen Sinne in Deutschland auf Bundesebene überhaupt nicht. Alle Einzelbeobachtungen Schefolds, die ja zutreffen, bringt man üblicherweise auf den Begriff einer informellen Großen Koalition bzw. in Teilbereichen sogar einer informellen All-Parteien-Koalition. Der Autor hätte hier seinen Terminus, zumal er den ganzen Beitrag in gewisser Weise einklammert, nicht ohne Erläuterung und vergleichende Hinweise verwenden sollen.

In dem im Herbst 1990 abgeschlossenen Beitrag „Reichsgründungstag“ vergleicht Schefold zunächst die Einigungsprozesse, die 1870/71 zum Kaiserreich bzw. 1990 zum wiedervereinigten Deutschland führten, und arbeitet ihren Charakter als von den Regierungen ausgehandelte Zusammenschlüsse heraus; Verfassungsgebung bzw. -änderung hatten diese nur nachzuvollziehen. Dementsprechend sieht er den „Tag der Deutschen Einheit“ am 3. Oktober auch in der Traditionslinie des „Reichsgründungstages“ (18. Januar). Der Weimarer Versuch, (zusätzlich) einen Verfassungstag zu feiern (11. August), konnte sich schon seinerzeit nicht recht durchsetzen. Das Diktum des FAZ-Mitherausgebers Fritz Ullrich Fack vom 1. September 1990: „Was am 3. Oktober zum alt-neuen staatlichen Gehäuse aller Deutschen zusammenwachsen wird, braucht weder eine neue Verfassung noch einen neuen Namen noch irgendwelche Volksabstimmungen“, versteht Schefold zutreffend als die „Leitidee des Einigungsvertrags“ (S. 289).

Dennoch spürt er, angeregt von den seinerzeitigen zivilgesellschaftlichen Bestrebungen, der Möglichkeit nach, die Verfassung des neuen gesamten Deutschlands ausdrücklich demokratisch zu legitimieren - im Unterschied zu dem 1949 nur „prekär“ (S. 290) demokratisch legitimierten, aber inzwischen akzeptierten Grundgesetz der alten Bundesrepublik -, und zwar zunächst durch Interpretation 
des durch seine Änderung 1990 eher verdunkelten Art. 146 GG $^{23}$. Wider den damaligen Mainstream, der sich ja bis heute behauptet hat, besteht Schefold auf der „Plausibilität direkt-demokratischer Verfahren der Verfassungsgebung“, ja seines Erachtens „zwingt die Neufassung des Art. 146 dazu, die Frage des Volksentscheids zumindest in Verfassungsfragen zu regeln“ (S. 292). Seine Warnung freilich vor Plebisziten, also einer „Entscheidung in einer konkreten Situation ohne normative Vorgabe“ (S. 291), erscheint angesichts einschlägiger Gedankenspiele in der gegenwärtigen Euro-Krise hochaktuell.

In dem Beitrag „,Von der Grundrechtsinterpretation zur Verfassungstheorie“, zuerst 2006 erschienen, untersucht Schefold für den Zeitraum von Mitte der sechziger bis Mitte der achtziger Jahre zunächst, ob und wie die Grundrechtsinterpretation „entscheidende Impulse erhalten und gegeben“ hat (S. 294). Dazu zieht er vor allem die Rechtsprechung des Bundesverfassungsgerichts heran, das die Entwicklung der Grundrechtsdogmatik - freilich auch schon in der Zeit davor - in hohem Maße gefördert habe. Dies hat jedoch Konsequenzen. Da sich die Rechtsprechung ,vor allem mit der konkreten Einzelinterpretation der Grundrechte auseinanderzusetzen" hat (S. 305) und dabei praktisch eher induktiv vorgeht, bewegt sie die Wissenschaft in erste Linie zum Versuch einer Systematisierung „unter weitgehendem Verzicht auf theoretische Begründung“ (S. 306 f.). Die Frage: „Welchem Zweck dient die Grundrechtsgewährleistung, und wie ordnet sie sich in das Regelungs- und Gestaltungsprogramm der Verfassung ein?“ (S. 307), bleibt so unbeantwortet, obwohl eine Grundrechtstheorie nötig wäre, weil konkrete Interpretationsergebnisse durchaus von ,theoretischen Annahmen über die Funktion der Grundrechte“" abhängen (S. 308).

Alsdann mustert Schefold kritisch fünf von Böckenförde vorgelegte theoretische Ansätze und versucht eine verfeinerte Systematisierung. Aus vier materiellen Prinzipien und drei methodischen Instrumenten entsteht so ein zwölfteiliges Systemraster. Im Folgenden versucht Schefold, „das grundrechtliche Schrifttum mit theoretischem Anspruch“ (S. 312) - Peter Häberle, Friedrich Müller, Peter Saladin, Niklas Luhmann, Helmut Ridder und Robert Alexy - einzuordnen. Anschließend erweitert Schefold die Perspektive auf das Verfassungsrecht insgesamt, jenseits der Zentrierung auf die Grundrechte. Hier skizziert er eine „Gegenbewegung gegen die Grundrechtseuphorie in der Form einer erneuerten Staatsmetaphysik“ (S. 318) - Ernst Forsthoff, Herbert Krüger - sowie auf der anderen Seite die in der Formel vom „,nichterfüllten Grundgesetz“ zusammenzufassenden Versuche, „,er Verfassung, über die konkret gewährleisteten Abwehrrechte und damit Schranken des Gesetzgebers hinaus, Grundsätze für die weitere 331). 
Ausgestaltung der Gesetzgebung zu entnehmen“ (S. 323). In diesem Sinne könne die Gesetzgebung der (ersten) großen wie der sozialliberalen Koalition (1966-69, 1969-82) „weitgehend als Tätigkeit der Verfassungsverwirklichung verstanden werden“" (S. 320).

Die Grundrechte - so ein weiterer Gedankengang - müßten in eine Verfassungstheorie eingebettet sein, doch dürfe man den Topos von der „Einheit der Verfassung" nicht überstrapazieren. Bei den Grundrechten, die unterschiedlichen Quellen und Einflüssen entstammten, müsse man nach „Garantieschichten“ differenzieren (S. 325); die Forderung nach einer einheitlichen Grundrechtstheorie führe zu einer unzulässigen Nivellierung. Wenn Grundrechte in einem Spannungsverhältnis zueinander stünden, bedürfe es eines Ausgleich im Wege der „praktischen Konkordanz“ (Konrad Hesse).

Abschließend greift Schefold unter der Zwischenüberschrift „Von der Verfassungstheorie zur Verfassungsgebung" noch etwas auf die Zeit nach seinem eigentlichen Untersuchungszeitraum aus und skizziert kurz die innovative Verfassungsrevision in Schleswig-Holstein 1989 und die Verfassungsdiskussion des folgenden Jahres im Zusammenhang der Wiedervereinigung. Daß sich bei letzterer die „Beitrittslösung“ durchsetzte, bedeutete, daß „die Verfassungsfrage im Sinne Isensees erledigt" schien - für 1990. Viele wichtige Themen der damaligen Diskussion - nicht zuletzt Formen der direkten Demokratie -, die auch in der Gemeinsamen Verfassungskommission auf keinen fruchtbaren Boden fielen, fanden in den folgenden Jahren „Widerhall“ (S. 332) auf der Landesebene, sei es bei der Verfassungsgebung in den neuen, sei es bei Verfassungsrevisionen in den alten Bundesländern.

Der Beitrag „Deutschland als Parteiendemokratie“ erschien zuerst 1999 in dem PVS-Sonderheft „50 Jahre Bundesrepublik Deutschland“. Als erstes erklärt Schefold die „Parteiendemokratie“ - er wählt, überzeugend, diesen zurückhaltenderen Begriff anstelle des umfassenderen Terminus „Parteienstaat“ (vgl. S. 340) - als „Verfassungsgrundsatz mit normativem Gehalt“ (S. 334). Nach der Vorgeschichte einer Abwehrhaltung von der Zeit des Deutschen Bundes (1832) bis noch zur Weimarer Reichsverfassung (Art. 130 Abs. 1) und nach der Perversion des Einparteienstaates der NSDAP begreift er Art. 21 GG als Ausdruck des 1949 bestehenden Bedürfnisses, „der rechtlichen Regelung der politischen Parteien eine Grundlage auf Verfassungsstufe zu geben“ (S. 338), eine Tendenz, die sich - nun positiv verstanden - bis zum Vertrag von Maastricht 1992 (Art. 138a EGV), ja inzwischen weiter bis zum Vertrag von Lissabon 2007 (Art. 10 Abs. 4) verfolgen läßt ${ }^{24}$. Im weiteren handelt Schefold über die Anwendungsbereiche der

24 Freilich hat sich in diesen 15 Jahren der Akzent verschoben: Sollten die Parteien 1992 dazu beitragen, „ein europäisches Bewußtsein“ herauszubilden und „,den politischen Wil- 
Parteiendemokratie, die Sicherung innerparteilicher Demokratie, die Auswirkungen auf die Parteienfinanzierung und über Parlamentarisches Parteienrecht als Aufgabe.

Das ist alles kenntnisreich und gediegen geschrieben, aber am interessantesten ist ein Absatz, bei dem man mit dem Autor streiten muß. Zum Unterthema „Parteiendemokratie und unmittelbare Demokratie" formuliert Schefold, es lasse „sich angesichts der Gewährleistung pluralistischer Mitwirkung der politischen Parteien gegen Entscheidungsmechanismen argumentieren, die diese Mitwirkung ausschalten, das Volk als Einheit zur Artikulation bringen können. Die Parteiendemokratie stellt sich Bestrebungen der unmittelbaren Demokratie entgegen und beruft sich auf den Grundsatz der innerparteilichen Demokratie." (S. 340). So mag es manchmal faktisch vorgetragen werden, aber so läßt sich, jedenfalls wissenschaftlich, durchaus nicht argumentieren. Der Zweifel entsteht schon methodisch. Schefold, der sich eingangs einem primär politikwissenschaftlichen Leserpublikum ausdrücklich als Verfassungsjurist vorstellt, bringt nämlich nach den zitierten Sätzen gerade keine verfassungsrechtliche Begründung, sondern verweist auf Ernst Fraenkels Bemerkung über die ,politisch [!] erträglichen Grenzen“" plebiszitärer Verfassungsinstitutionen - das ist Politikwissenschaft und dessen Prüffrage, ob die Wähler noch überzeugt seien, „daß sie in ihren Parteien Gebilde besitzen, die ihre Wünsche und Ansichten ausreichend vertreten“ (S. 341) - darauf könnte die Soziologie eine Antwort geben.

Die vermißte verfassungsrechtliche Argumentation müßte etwa lauten:

- Erstens: Wenn man von der Allgemeinheit der „Parteiendemokratie“ zurückgeht auf den Verfassungstext, dann ,wirken“ nach Art. 21 Abs. 1 Satz 1 GG die Parteien bei der politischen Willensbildung des Volkes „mit“. Dies schließt eine Monopolstellung aus. Man darf nicht lesen: Die politischen Willensbildung des Volkes vollzieht sich durch die Parteien.

- Zweitens: Art. 20 Abs. 2 Satz 2 GG, als Staatsfundamentalnorm durch die Ewigkeitsklausel des Art. 79 Abs. 3 GG geschützt, bestimmt, daß das Volk die Staatsgewalt - außer durch die besonderen Organe - in ,Wahlen und Abstimmungen“ ausübt. Mag dies, was die „Abstimmungen“ angeht, auch nur (aber immerhin) eine ,,plebiszitäre Strukturoption“ $\operatorname{sein}^{25}$, so sei doch festgehalten, daß das Grundgesetz bei Territorialplebisziten durchaus Abstimmungen sowie Volksbegehren und Volksentscheid kennt bzw. kannte (vgl. Art. 29 [Fassung 1949] Abs. 4 Satz 2, 118, 118a GG).

len der Bürger“ zum Ausdruck zu bringen, ist das Adjektiv „politisch“ seitdem gewandert: Jetzt (2007) ist von der Herausbildung ,eines europäischen politischen Bewußtseins“" und dem „Ausdruck des Willens der Bürgerinnen und Bürger“ die Rede.

Vgl. Otmar Jung, Grundgesetz und Volksentscheid. Gründe und Reichweite der Entscheidungen des Parlamentarischen Rats gegen Formen direkter Demokratie, 1994, S. 313-322. 
- Drittens: Schefold betont beim Thema Parteienfinanzierung, daß „nur eine Optimierung zwischen den gegenläufigen Prinzipien in Betracht" komme (S. 349) - warum dann bei der direkten Demokratie diese harte Entgegensetzung? Zumal gar nicht ausgemacht ist, daß eine solche überhaupt besteht. Man könnte auch mit Ulrich K. Preuß argumentieren: ,Zum Prozeß der politischen Willensbildung gehören Wahlen und Abstimmungen, weil in ihnen das Volk selbst unmittelbar uno actu als Staatsvolk und als politische Gesellschaft handelt.“ Ja, „Wahlen und Abstimmungen“ sind sogar „ein wesentliches Element der politischen Willensbildung, so daß eine dauerhafte Nichtbeteiligung an ihnen zum Verlust der Parteieigenschaft führt ${ }^{\text {‘26. }}$.

Endlich wäre hier ein sozialwissenschaftlicher Blick auf die Praxis direkter Demokratie in Deutschland hilfreich, der zeigte, daß speziell Volksbegehren zwar nicht, wie es oft abschätzig hieß, bloß „Parteibegehren“ sind, aber in keinem parteifreien Raum stattfinden. Angesichts des grundsätzlich oppositionellen Charakters der Volksgesetzgebung positionieren sich die Parteien schon beim Volksbegehren, und vor dem Volksentscheid am Ende des Verfahrens „mischen“ sie mit wahlkampfähnlichen Abstimmungskämpfen „mit“. Fraenkels Diktum - den ich nun meinerseits zitiere -, daß ein Plebiszit unter einem parlamentarischen Regierungssystem „bestenfalls ... das Abhalten eines außerparlamentarischen ParteiExerzierens" sein könne ${ }^{27}$, unterschätzte gewiß die Vitalität der zivilgesellschaftlichen Kräfte. Aber wenn sich Schefold vier Jahrzehnte später angesichts des Aufschwungs der direkten Demokratie nach 1989 auf Landesebene um die grundgesetzlich geschützte „Mitwirkung“ der Parteien an der politischen Willensbildung sorgt, fällt er sozusagen ins andere Extrem und unterschätzt die Adaptionsfähigkeit von Parteien an neue Situationen.

Am Ende des Absatzes behauptet Schefold, jener Aufschwung habe „die Parteiendemokratie geschwächt. Aber auf Bundesebene hat sich der Angriff unmittelbar demokratischer Institutionen nicht durchgesetzt, und die Abwehr gegen die Neuregelungen dauert an“ (S. 341). Hier ist der Ausdruck „Angriff“ zu bedauern. Wenn Bürger sich dafür engagieren, daß im ordentlichen Wege der Verfassungsänderung die plebiszitäre Strukturoption des Grundgesetzes zu praktisch nutzbarem Verfassungsrecht aktualisiert wird und damit der deutsche Gesamtstaat ebenso zu einer ,gemischten“ Demokratie wird wie alle seine Länder (und Kommunen) - ein Versuch, wie er in der Gemeinsamen Verfassungskommission 1992/93 ohne Erfolg unternommen worden war (und von der rot-grünen Koaliti-

26 Ulrich K. Preuß, Alternativkommentar zum Grundgesetz, 2. Aufl. 1989, Art. 21 Abs. 1, 3 Rn. 26.

27 Vgl. Ernst Fraenkel, Die repräsentative und die plebiszitäre Komponente im demokratischen Verfassungsstaat, 1958 (Recht und Staat in Geschichte und Gegenwart H. 219/220), S. 28 f. 
on im Bundestag 2002 wiederholt werden sollte) -, dann erscheint ein Begriff nicht korrekt, der mit Feindseligkeit, Gewalt und, wenn auch noch „Abwehr“ hinzukommt, letztlich mit Krieg konnotiert ist, zumal dieser „Angriff“ typischerweise ,von außen“ vorgetragen wird. Hier aber handeln Bürger dieses Staates, die seine demokratische Ordnung im Wege der Reform verbessern wollen mag ihr Konzept auch nicht jedermann überzeugen. Sowenig diese reformeifrigen Bürger ,angreifen“, sowenig tun es - um Schefolds Formulierung wortwörtlich aufzunehmen - die direktdemokratischen Institutionen selbst. Alle einschlägigen Projekte firmieren als eine „Ergänzung“ der repräsentativen Demokratie des Grundgesetzes durch direktdemokratische Elemente. Soweit aus einer solchen Ergänzung Spannungen entstehen sollten, wären sie nach dem auch sonst von Schefold befürworteten Konzept der „praktischen Konkordanz“ bei widerstreitenden Verfassungspositionen auszugleichen ${ }^{28}$.

In einer Normenhierarchie darauf zu achten, daß die jeweils unteren Rechtsvorschriften den oberen entsprechen, ist selbstverständliche Aufgabe der Rechtsanwender. Diese wird in der Moderne schwierig durch die Ausdifferenzierung der Rechtsquellen und durch die Vielzahl der Ebenen, auf denen dies geschieht: vom Völkerrecht über das Recht der Europäischen Union, das Recht der Nationalstaaten und ihrer föderalen bzw. regionalen Gliederungen bis zum Recht der Gemeinden. In seinem Beitrag ,,Verfassungskonforme Auslegung zwischen Italien und Deutschland", zuerst 2008 erschienen, beschäftigt sich Schefold mit einem Hauptanwendungsfall dieser Aufgabe: in der Verfassungsgerichtsbarkeit, wo die beiden Rechtsordnungen eng verwandt sind. Nach Darlegung der methodischen Grundlagen arbeitet Schefold die normativen Merkmale der deutschen Verfassungsgerichtsbarkeit in Bezug auf Normenkontrolle und verfassungskonforme Auslegung heraus und stellt vier wichtige Unterschiede zu Italien fest:

- In Deutschland obliegt die Verfassungsmäßigkeitskontrolle in erster Linie dem Fallrichter; dies schließt die Aufgabe der verfassungskonformen Auslegung des Gesetzes ein. Nur verwerfen darf der Fallrichter nicht; dann hat er im Wege der Richtervorlage das Bundesverfassungsgericht anzurufen. Hingegen entscheidet in Italien der Verfassungsgerichtshof allein über die Frage der Verfassungsmäßigkeit von Gesetzen.

- Das Bundesverfassungsgericht hat sein Verwerfungsmonopol auf die unter der Geltung des Grundgesetzes entstandenen Gesetze beschränkt; vorkonstitutionelle Gesetze hat der Fallrichter selbst auf das Grundgesetz auszurichten. Der italienische Verfassungsgerichtshof dagegen hat „auch gegenüber gerichtshof dies in vorbildlicher Weise tun, Urt. v. 11.7.2002, LVerfGE 13, 315. 
vorkonstitutionellen Gesetzen ein Monopol der Verwerfungskompetenz für sich in Anspruch genommen" (S. 361).

- In Deutschland haben Normenkontrollentscheidungen des Bundesverfassungsgerichts Bindungswirkung und Gesetzeskraft, gleichviel, ob die Verfassungsmäßigkeit des geprüften Gesetzes verneint oder bejaht wird. In Italien beschränken sich diese Wirkungen auf die normverwerfenden Entscheidungen des Verfassungsgerichtshofs.

- Zum Bundesverfassungsgericht kann man Verfassungsbeschwerde erheben, während die italienische Rechtsordnung diesen Behelf nicht kennt. Spannend wird nun der Blick auf die Folgen dieses unterschiedlichen rechtlichen Instrumentariums unter der thematischen Leitperspektive. Die Ausgestaltung der Verfassungsgerichtsbarkeit in Deutschland „,begünstigt“ nach Schefold die verfassungskonforme Auslegung (S. 365). Gleichwohl ist deren Bedeutung, über die Jahrzehnte betrachtet, zurückgegangen. Bezweckte man mit ihr ursprünglich die „Vermeidung der Nichtigerklärung“ (S. 366), hilft sich das Bundesverfassungsgericht inzwischen eher mit der „Verfassungswidrigerklärung unter vorläufiger (befristeter) Fortgeltung“ (S. 367). Durch die Verfassungsbeschwerde, die 90 Prozent aller Fälle ausmacht, wird eine Fülle von Rechtsproblemen nach Karlsruhe gebracht, aus der sich das Bundesverfassungsgericht das Wichtigste zur Entscheidung heraussuchen kann, um so die Falljudikatur einheitlich zu kontrollieren. Damit hat das Bundesverfassungsgericht inzwischen „die Funktion eines obersten Bundesgerichts“ (S. 368), wie es ursprünglich als eigene Institution vorgesehen war (Art. 95 GG a.F.). Es entscheidet, wie man in der Literatur formuliert hat, als „subsidiärer Superrevisor“ (vgl. S. 368). Insgesamt aber kommt Schefold zu dem Ergebnis, „daß die maßgebliche Rolle des Fallrichters einerseits, verbunden mit der generellen Kontrolle des Bundesverfassungsgerichts über alle Gerichtsbarkeiten mittels der Entscheidung über Verfassungsbeschwerden andererseits ein Gleichgewicht zwischen der Rechtsentwicklung, die allen Richtern obliegt, und deren Koordination und Kontrolle durch die Verfassungsgerichtsbarkeit bewirkt"“ (S. 369).

Noch spannender ist die rechtstatsächliche Situation in Italien. Die verfassungskonforme Auslegung mit dem Ziel, den gesetzlosen Zustand zu vermeiden, „bestimmt anscheinend nach wie vor die Rechtsprechung“ (S. 370). Das Monopol des Verfassungsgerichtshofs, verfassungskonform auszulegen, ließ sich aber praktisch nicht durchhalten und führte zu erheblichen Rückständen anhängiger Verfahren, so daß „nach der Praxis und der neueren Lehre“ inzwischen „auch in Italien, wie in Deutschland, die Zuständigkeit zur verfassungskonformen Auslegung auf die Fallrichter und auf das Verfassungsgericht aufgeteilt ist“" (S. 371). Ferner hat die Fülle der Normenkontrollvorlagen in Italien, zehnmal so viele wie in Deutschland, auch dem Verfassungsgerichtshof hinreichend Gelegenheit ge- 
geben, „zu unterschiedlich entschiedenen Fragen Stellung zu nehmen und die von ih $[\mathrm{m}]$ postulierte Auslegung durchzusetzen“ (S. 372).

Im Ergebnis - so Schefold - nähert sich „die italienische Praxis der deutschen an. Die fehlende Bindungswirkung normbestätigender Entscheidungen und das Fehlen der Verfassungsbeschwerde werden durch die Zahl und Intensität der Normenkontrollentscheidungen, vor allem der verfassungskonformen Auslegungen ausgeglichen, und im Fall gravierender Divergenzen steht nach wie vor das Instrument der Verfassungswidrigerklärung zur Verfügung.“ (S. 372). Es verbleibt ein gewichtiger Unterschied: Während in Deutschland außer einer günstigeren prozessualen Lage auch die Position als ,de facto oberstes Gericht und Kontrollorgan über die gesamte Rechtsprechung“ (S. 365) dem Bundesverfassungsgericht den eindeutigen Vorrang sichert, muß in Italien der Verfassungsgerichtshof, prozessual ohnehin schlechter gestellt, mit einem starken Gegenspieler in Gestalt des Kassationsgerichtshofs zurecht kommen (vgl. S. 373).

„Mehr Demokratie und Probleme direkter Demokratie in Deutschland“, erschien $2011^{29}$ und ist damit der jüngste hier ausgewählte Aufsatz. Geschrieben für die Roland Geitmann gewidmete sogenannte „Kuratoriums-Broschüre“ des Vereins Mehr Demokratie e.V., ist es ein wichtiger - und nicht zuletzt persönlicher - Text. Eingangs reflektiert Schefold die Ambivalenz des Themas: Einerseits ist direkte Demokratie in Deutschland Gegenstand wissenschaftlicher Diskussion und hat ,zu reicher wissenschaftlicher Auseinandersetzung Anlaß gegeben, von zeitgeschichtlichen, politikwissenschaftlichen und juristischen bis zu politisch-ökonomischen Beitragen“" (S. 375). Andererseits ist direkte Demokratie Gegenstand des politischen Streits, der Parteien, Parlamente und Verfassungsgerichte beschäftigt. Ähnliches gelte für das hier besonders interessierende Kuratorium für Mehr Demokratie, ein Gremium des gleichnamigen Vereins: Einerseits vereinen seine Mitglieder ,umfangreichen und gründlichen Sachverstand“; „die daraus resultierende fachliche Kompetenz ist beeindruckend“ (S. 375). Andererseits ist das Kuratorium heterogen, ob man das politische bzw. parteipolitische Engagement, die wirtschaftlichen Interessenbindungen oder die „manchmal gegensätzlichen wissenschaftlich-theoretischen Ausgangspositionen" betrachtet (S. 375). Diese Analyse ist richtig, man vermißt aber eine Klarstellung, daß Heterogenität nicht in schlechter deutscher Tradition als „Zerrissenheit“ negativ ver-

In: Hermann K. Heußner/Roman Huber/Otmar Jung (Hrsg.), Das Kuratorium für Mehr Demokratie. Roland Geitmann zum 70. Geburtstag, 2011, S. 58-65. Dort ist dieser Text in der Tat „schwer greifbar“ in einem modernen Sinne. Die in kleiner Auflage erschienene Broschüre ist auch eineinviertel Jahre nach ihrem Erscheinen im Karlsruher Virtuellen Katalog nicht nachweisbar (letzter Zugriff 21.7.2012). Der Kundige, der unter mehrdemokratie.de nachschaut, kann sich die 75 Seiten aber leicht herunterladen und ausdrucken (http://www.mehr-demokratie.de/kuratorium.html, Zugriff 21.7.2012). 
standen werden darf. Umgekehrt ist diese Vielfalt, die Nicht-Festlegung auf engere politische, wirtschaftliche oder wissenschaftliche Positionen eine Chance, ja geradezu die Voraussetzung der erreichten Wirksamkeit des Kuratoriums wie des ganzen Vereins.

Im folgenden Gedankenschritt stellt Schefold seine Beziehung zu Mehr Demokratie e.V. klar, aber darüber hinaus zu den politischen Debatten über direkte Demokratie in Deutschland überhaupt. Die Kernaussagen dazu, gipfelnd in dem Begriff des „Außenstehenden“, wurden bereits am Anfang dieser Besprechung referiert. Hier sei nur betont, daß der Abstand zu den Dingen, der Blick ,von außen“, gewiß Schefolds Urteil zugute kommt, sei es über die einschlägigen Debatten in Deutschland, sei es über diesen Verein und sein Kuratorium.

Als nächstes versucht Schefold, die „Bedeutung“ (S. 377) von Mehr Demokratie e.V. zu beschreiben und führt dazu zuerst eine Reihe von Verdiensten auf:

- Der Verein habe die neuere Diskussion der direkten Demokratie, verstanden als ,gemischte Demokratie“, vorangebracht (S. 377);

- er habe den zutreffenden Befund, daß die „Abstimmungen“, ebenso wie die Wahlen, ,notwendige Ausprägung des in Art. 20 [GG] verankerten Demokratieprinzips" seien, in Erinnerung gerufen (S. 378);

- er habe mit zahlreichen Kampagnen „entscheidend“ dazu beigetragen, daß Elemente direkter Demokratie auf der Kommunal- und Landesebene den Durchbruch schafften (S. 378); auch bei der Verfahrensgestaltung sei sein Einfluß ,augenfällig“ (S. 382);

- ebenso habe er mit Erfolg dafür gekämpft, daß in den letzten Jahren in den meisten Ländern die Verfahrenshürden „entscheidend gesenkt“ wurden (S. $385)$;

- schließlich habe er auf Bundesebene „das Problem zumindest ins Gespräch gebracht" und immerhin 2002 eine einfache Pro-Mehrheit im Bundestag erreicht $^{30}$, auch wenn diese nicht für eine Verfassungsänderung genügte (S. 378).

Alsdann geht Schefold auf einige kritische Punkte ein, die freilich weniger den Verein als solchen, als vielmehr manche in diesem Kreis bezogene Position betreffen. Aus geht er dabei von der Maxime, daß es bei der Volksgesetzgebung „nicht nur um ein Ja oder Nein, sondern vor allem um das Maß, die Ausgestaltung, die Modalitäten, die Rahmenbedingungen“ gehe (S. 379). Hier schlägt er

30 Wichtig ist dabei das Engagement des Grünen-Abgeordneten Gerald Häfner, zugleich Mitglied des Bundesvorstands von Mehr Demokratie e.V., vgl. Otmar Jung, Direkte Demokratie. Die Angst der politischen Klasse vor dem Volk: Fortschritte und Rückschritte in den letzten 15 Jahren in Deutschland, in: Defizite in Staat und Verwaltung. Beiträge auf der 10. Speyerer Demokratietagung vom 25. und 26. Oktober 2007 an der Deutschen Hochschule für Verwaltungswissenschaften Speyer, hrsg. von Hans Herbert v. Arnim, 2010 (Schriftenreihe der Hochschule Speyer Bd. 203), S. 105-171 (130-136). 
gewissermaßen drei Pflöcke ein, die allesamt dem Referendum gelten, das er „ins Hintertreffen geraten“ sieht (S. 384):

- Das obligatorische Verfassungsreferendum sei das klassische Mittel, um am Ende eines Verfassungsgebungs- oder -änderungsprozesses „die Übereinstimmung von dessen Ergebnis mit dem Volkswillen sicherzustellen“. Gerade ,zur Volksgesetzgebung gehört als schwerlich verzichtbare Voraussetzung, daß sie auf einer Grundlage beruht, die vom Volk beschlossen worden ist" (S. 380) $)^{31}$.

- Das fakultative Gesetzesreferendum sei das geeignete Mittel, um anstelle der Rousseauschen Utopie, nach der alle Gesetze der Bestätigung des Volkes unterstehen müßten, „die relevanten, streitigen und alternativen Lösungen zugänglichen Fragen zu ermitteln und den Volksentscheid darauf zu konzentrieren“" (S. 381) . $^{32}$

- Auch auf europäischer Ebene seien Referenden das richtige Mittel, um das offenkundige Demokratiedefizit auszugleichen. Dabei solle man mit der Forderung nach einem konsultativen Referendum beginnen, das in einem späteren Schritt zu „einem echten Verfassungsreferendum“ ausgebaut werden könnte (S. 383).

Abschließend stellt Schefold noch einige Maximen sozusagen für die Gesamtdiskussion in Deutschland auf:

- Für die Direktwahl der Exekutivspitzen, etwa der Bürgermeister, könne man durchaus die „Aktivierung lokaler Demokratie“ als Argument anführen; aber diese verstärke jedenfalls nicht die Kontrolle der Bürger über die Tätigkeit der Gemeindevertreter. „Die Frage gehört daher in einen besonderen $\mathrm{Zu}$ sammenhang" (S. 379), und nicht zur sachunmittelbaren Demokratie.

- Schefold wendet sich gegen alle Surrogat-Argumentationen. „Das Demokratiedefizit auf europäischer Ebene ist zu beheben, aber durch Vorkehrungen

31 Der Beitrag ist, dem seinerzeitigen Anlaß angemessen, kürzer als etwa der vorher abgedruckte. So sind auch einige Positionen nur angetippt, ohne alle weiteren Verästelungen zu behandeln. Daß das obligatorische Verfassungsreferendum ,einer allzu häufigen Verfassungsänderung“ vorbeuge (S. 380) - beispielsweise -, ist nur auf den ersten Blick überzeugend. Bedenkt man die Konterstrategien der repräsentativ-demokratischen Institutionen, insbesondere daß die Regierungen ganze Pakete schnüren, wenn schließlich doch einmal die Verfassung geändert wird, könnte sich jene Vorbeugung in der demokratischen Bilanz als illusionär erweisen. Vgl. Otmar Jung, Regieren mit dem obligatorischen Verfassungsreferendum: Wirkung, Konterstrategie, Nutzungsversuche und Umgangsweise, in: ZParl 36 (2005), S. 161-187 (172-176).

32 Siehe wie vor. Daß ,gegen mißliebige Gesetze“ auch das Volksbegehren zur Verfügung steht (S. 381), ist richtig. Nur erscheint die in Deutschland übliche Konstellation, daß die Landesverfassungen den Opponenten das fakultative Gesetzesreferendum vorenthalten und sie dafür auf den Weg der Volksgesetzgebung verweisen, wegen des ungleich größeren Aufwands in doppelter Hinsicht unfair. Vgl. dazu näher Otmar Jung, Die Reform der direkten Demokratie in Berlin 2006 (Teil II), in diesem Jahrbuch, S. 303-281 (303 f.). 
auf europäischer Ebene, die auf der Ebene der Mitgliedstaaten ebenso wenig ersetzt werden können, wie Volksentscheide in den Ländern den fehlenden Volksentscheid auf Bundesebene ersetzen können.“ (S. 384). Sein Schluß: „Daß der Ruf nach einem Volksentscheid über das deutsche Zustimmungsgesetz $^{33}$ nicht erhört worden ist, scheint mir daher sachlich gerechtfertigt" (S. 384), überzeugt freilich nicht. Solange auf der europäischen Ebene die gebotenen direktdemokratischen Instrumente noch gar nicht zur Verfügung stehen, erscheint Schefolds Argumentation hier puristisch.

- Das Finanztabu, dieses zweifelhafte deutsche Alleinstellungsmerkmal, erklärt Schefold aus einem spezifisch obrigkeitsstaatlichen Verständnis: „Faßt man Volksbegehren als Aufbegehren der Bürger gegen den bestehenden Rechtszustand auf, so mögen die Staatsfinanzen als unantastbar, mag der Schutz der bestehenden Ordnung als prioritär qualifiziert werden ${ }^{34}$. Aber wenn - und weil - es um die Verifizierung einer Vertretung des Volkes geht ..., gehört dazu die Regelung finanzieller Fragen ... warum soll das Abgabensystem, warum die Höhe der Bezüge aus öffentlichen Kassen - gerade auch für Abgeordnete und Beamte -, das sonstige Ausgabenverhalten der öffentlichen Hände nicht dem Volksentscheid unterstellt werden? ... Volksgesetzgebung hat die Funktion, insofern als Kontrollmittel zu dienen. Gerade gegenüber privilegierendem und allzu ausgabenfreudigem Verhalten kann sie, auch nach schweizerischen Erfahrungen, von Nutzen sein - Stichwort: Ausgabenbremse“" (S. 386 f.).

- Es bleibt das Problem der Verfassungsmäßigkeitskontrolle von Volksbegehren, das Schefold Ende 2009, als er den Beitrag abschloß - gerade den Volksentscheid zur Aufnahme eines Minarettverbots in die Schweizerische Bundesverfassung vor Augen -, besonders beschäftigte. Für diesen speziellen Fall eines verfassungsändernden Volksgesetzgebungs-Projekts erscheint ihm die Festlegung eines änderungsfesten Kerns in Art. 79 Abs. 3 GG stabiler als die ,vorsichtigere“ Regelung des Art. 139 Abs. 3 BV. Für den Fall einer entsprechenden Ergänzung des Grundgesetzes spricht er sich aber ,namentlich für höhere Unterschriften- und Zustimmungsquoren, vielleicht auch

33 Nicht ganz eindeutig, ob das Zustimmungsgesetz zum Vertrag von Lissabon 2007 oder jenes zum Vertrag von Maastricht 1992 gemeint ist.

34 Diesen falschen Ansatz, den die direkte Demokratie in Deutschland schon 1919 genommen hatte, hatte Karl Neumayer, ebenfalls in beiden Rechtsordnungen zu Hause, schon vor vier Jahrzehnten mit Verve kritisiert: Die Volksrechte wurden „nicht als Ausdruck der obersten Volkssouveränität verstanden“, sondern „,als artfremder Eingriff in die eingerichtete staatliche Ordnung“ qualifiziert. ,Wer die Initiative ergreift, der steht auf wider den Staat, ja gegen sein eigenes Volk, aufs beste organisiert und legitim verkörpert in Volksvertretung und Regierung." So ders., Betrachtungen zum Volksinitiativverfahren im Staatsrecht der Länder des deutschen Sprachgebietes ausserhalb der Schweiz, in: Mélanges Marcel Bridel, 1968, S. 321-345 (341 ff.). 
für besonders qualifizierte Vorprüfungsverfahren“ aus. Freilich - diese Situation liegt für Deutschland ,,in weiter Ferne“. Und so schließt der realistische Beobachter mit einer theologischen Utopie: „Eia wär'n wir da!“ (S. 387).

\section{Zur Lage der Stadtstaaten}

Dieses Kapitel, seitens des Wahl-Bremers Schefold natürlich unerläßlich, ist das schmalste; es umfaßt nur zwei Texte. Der erste Beitrag „Der Stadtstaat als demokratische Zukunftsperspektive" beruht auf einem Vortrag und wurde 1994 in dem Sammelband „Lokale Demokratie auf dem Prüfstand“ veröffentlicht. Schefold geht dabei aus von dem damals gerade ergangenen zweiten Finanzausgleichs-Urteil des Bundesverfassungsgerichts und von dem offenkundigen Befund der „Konflikte, Doppelspurigkeiten, Reibungsverluste“ in den Beziehungen Bremens zu seinem Umland. Sein Ziel ist es jedoch, „die Frage der Legitimation von Stadtstaatlichkeit und speziell des Landes Bremen etwas grundsätzlicher“ zu beantworten.

Dazu schildert er zunächst „die Bedeutung der Stadtstaaten“ im deutschen föderativen System und „den Stellenwert des Neugliederungsgedankens“ (S. 390). Er arbeitet heraus, wie die sogenannten Freien (Reichs-)Städte schon im Heiligen Römischen Reich Deutscher Nation eine „Alternative zum monarchischen Prinzip“ bildeten (S. 395). In ihnen verkörperte sich „eine demokratische Perspektive“ - „im Gegensatz zum monarchischen Obrigkeitsstaat“ (S. 397), wie sich besonders sinnfällig 1848 zeigte: „Während die März-Revolution in den meisten monarchischen Staaten Deutschlands zur echten Revolution wird“ und das „Verfassungsgebungswerk meist am monarchischen Widerstand scheitert, führt die März-Bewegung in Bremen zur Wahl einer verfassunggebenden Bürgerschaft nach gleichem Wahlrecht und ... zur Vereinbarung der am 21.3.1849 verkündeten Verfassung ... Die Stadtrepublik trägt die Wurzel der Demokratisierung in sich und läßt sie sich entfalten“ (S. 396).

Diese Sinngebung hatte sich freilich mit der Novemberrevolution 1918 und dem Übergang zur demokratischen Republik erschöpft. Hugo Preuß, der Schöpfer der Weimarer Reichsverfassung, nahm bei seinen Neugliederungsplänen für das Reich denn auch auf die Kleinstaaten keine Rücksicht. Der NeugliederungsArtikel 18 WRV, „damals eine absolute Neuerung“ (S. 391), drückte dieses neue Denken aus. Freilich scheiterte die geplante Neugliederung nicht an durchgreifenden Gegenargumenten, sondern einfach am Beharrungsvermögen, ja am ,erbitterten Widerstand“ der Länder, auch mit nunmehr freistaatlicher Verfassung, ein politischer Kampf, der sich nach 1949 mit dem Neugliederungsartikel 29 GG wiederholte. Als Ergebnis ist ein großer Sieg des Status quo zu vermelden; die 
Länder - große und kleine, leistungsfähig oder stützungsbedürftig, Flächen- oder Stadtstaat - sind rechtlich gut abgesichert.

Nur - „entspricht dieser starken Stellung aller Länder und damit auch der Stadtstaaten eine fortbestehende inhaltliche Sinnhaftigkeit stadtstaatlichen Verfassungsrechts?“ (S. 397). Dieser Frage geht Schefold nun speziell für Bremen nach, wobei er zunächst der ,doch recht intensiven Kontinuität bremischer Verfassungsstrukturen“ von 1849 über 1920 bis 1947 gedenkt (S. 397) und den Charakter der geltenden Landesverfassung als vorgrundgesetzlich betont. Im einzelnen erörtert er einige „Kontinuitätselemente des traditionellen bremischen Verfassungsrechts“, die ihm ,erhaltens- oder zumindest fortbildungswürdig“ erscheinen (S. 399):

- Die Teilidentität der Organe in Land und Stadt Bremen - eine „elegante Lösung“" (S. 399),

- die Selbstverwaltungshoheit Bremerhavens, „der zweiten Stadt des Landes“ (S. 400),

- den Senat als Kollegium ${ }^{35}$,

- die Deputationen und nicht zuletzt

- die „gemischte Demokratie“, welche die repräsentativ-demokratische Grundstruktur durch Elemente direkter Demokratie ergänzt - eine Tradition, die in Bremen bis auf die Verfassung von 1849 zurückgeht, die ihrerseits sich an schweizerischen Vorbildern orientierte ${ }^{36}$.

Dies belegt für Schefold eine Grundthese: „Der Stadtstaat in seiner Verfassung hat Zukunftsperspektive, wenn er seine Eigenart bejaht und dazu steht, sich demokratisch - mit dem Plus an Demokratie, das örtlich besser möglich ist als im Flächenstaat - konstituiert und im übrigen für Zusammenarbeit und Entwicklung offen ist" (S. 402).

Aber trotz „dieses Ja zu einer möglichen und wünschbaren Verfassungskultur des Stadtstaats“" (S. 402) sind da doch immer noch die eingangs genannten StadtUmland-Probleme. Hier plädiert Schefold für die „Kooperation als Alternative zur Neugliederung“ (S. 403), wofür er auch auf einen bremisch-preußischen Staatsvertrag ,über eine Gemeinschaftsarbeit“ von 1930 verweist (S. 392). Drei Jahrzehnte später ging es um die Gemeinsame Landesplanung Bremen/Nieder-

35 Eine Stärkung des Präsidenten des Senats hält Schefold „den Aufgaben eines Stadtstaats für weniger adäquat" (S. 401). Man erinnere sich, in welch unbefangener Machtpolitik die politische Elite Berlins 2006 vom stadtstaatlichen Senatsprinzip zum vom Bund und den Flächenländern her bekannten Kanzlerprinzip übergehen sollte, vgl. Otmar Jung, Die Reform der direkten Demokratie in Berlin 2006 (Teil I), in: JdD 3 (2011), S. 259-294 (263-272).

36 Vgl. Otmar Jung, „Ein Steckenpferd der Opposition“? Einflüsse schweizerischer direktdemokratischer Verfahren auf die Bremische Verfassung von 1849, in: Bremisches Jahrbuch 77 (1998), S. 99-124. 
sachsen in einem Gebiet mit zwei Millionen Einwohnern bzw. deren rechtliche Weiterentwicklung. Freilich bleibt, wie Schefold sieht, „die Institutionalisierung grenzüberschreitender Regionalplanung ein schweres und dennoch zerbrechliches Werk" (S. 394).

$\mathrm{Da} ß$ diese aus 40 Jahren eines Wissenschaftlerlebens ausgewählten Beiträge Spuren ihres Alters tragen, gehört zum Konzept des Genres Aufsatzsammlung. Und daß Schefold seine Texte nicht aktualisieren, d.h. neu schreiben wollte (Vorbemerkung, S. 12), ist gut zu verstehen. Freilich zeigt sich gerade bei diesem Beitrag eine gewisse Spannung zwischen jenen verfassungsgeschichtlichen Partien, die weiterhin schlechterdings gültig sind, und anderen, seinerzeit aktuellen Ausführungen, die bloß Momentaufnahmen der ersten Hälfte der 90er Jahre waren. Insofern wäre hier ein „Nachwort 2012“ wünschenswert gewesen, das den mühsamen Weg zu einem Verband Region Bremen weitergezeichnet hätte, der, obwohl seitens der Stadt Bremen ein hohes Interesse an ihm besteht, bis heute noch nicht Wirklichkeit geworden ist.

Der zweite Beitrag „Hundertfünfzig Jahre Bremische Verfassung. Vom Verfassungskonsens des 8. März 1849 zur Aufgabe bremischer Verfassungspolitik“, zunächst als Vortrag am Jubiläum gehalten, erschien (überarbeitet) 2000 im ersten Band des neuen „Jahrbuchs der Juristischen Gesellschaft Bremen“. Eingangs ruft Schefold die Umstände der Entstehung und die Atmosphäre der Promulgation jener Verfassung in Erinnerung, mit der „eine neue Epoche, die des Verfassungsstaats, für Bremen eingeleitet wurde“ (S. 407). Zwar wurde sie in Ausführung des sogenannten Bundesreaktionsbeschlusses von 1851 schon nach fünf Jahren abgelöst und blieb insofern „Episode“; dennoch hält Schefold hier das ,historische Verdienst Bremens“ fest: Diese Verfassung von 1849 könne „eine Modellfunktion für Deutschland beanspruchen, wie die demokratische Bewegung von 1848 in Verfassungspraxis umgesetzt werden konnte" (S. 409).

Vom Inhalt der Verfassung hebt Schefold die „Integration aller Bürger“, ihre „Trägerschaft der Staatsgewalt“ hervor, behandelt die Grundrechte, welche „die Verfassungskultur des Liberalismus spiegelt[en]“ (S. 411), und unterstreicht die „ganz spezifische, eigenständige Form der Gewaltenverschränkung“, die hier verfaßt wurde: „Senat und Bürgerschaft werden zur Zusammenarbeit gezwungen, aber dabei durch checks and balances gemäßigt" (S. 412).

Besonders interessant erscheint die Lösung der Verfassung für den Fall, daß Senat und Bürgerschaft nicht zu einem übereinstimmenden Beschluß gelangen. Dann wird nach Art. 3, § 6 , dieser Gegenstand an die Gesammtheit der Staatsbürger zur Entscheidung überwiesen“ (S. 414): ein arbitratorischer Volksentscheid also, dem auch noch ein Volksveto für den Fall der Verfassungsänderung folgt, beides wohl nach Vorbildern schweizerischer Kantone eingeführt. Schefold rühmt diesen Konfliktlösungsmechanismus: Für 1848 war er „,von radikaler 
Neuartigkeit; denn Ähnliches hatte es bis dahin in Deutschland nicht, in Frankreich, den USA und der Schweiz nur in Ansätzen gegeben“ (S. 414). Daß es in den wenigen Jahren der Geltung der Verfassung nicht zu Volksabstimmungen kam, liegt auf der Hand. Aber es sind bereits Fälle der Vorwirkung dieser Verfahren nachweisbar, was Schefold mit schweizerischem Wissen einordnet: „Volksentscheide als solche sind in der repräsentativen Demokratie ein Sonderfall; aber die Möglichkeit, sie herbeizuführen, bestimmt, verändert und rationalisiert den Charakter des Repräsentationsprozesses“" (S. 415).

Aus alldem leitet Schefold „,ünf Folgerungen für aktuelle Aufgaben bremischer Verfassungspolitik“ ab (S. 418), die freilich zumeist auf Kritik an Fehlentwicklungen bzw. Warnungen vor solchen hinauslaufen. Beispiel direkte Demokratie: Die Konzeption der geltenden Verfassung von 1947 sah das obligatorische Verfassungsreferendum vor, allerdings „mit der pragmatisch sinnvollen Ausnahmeklausel“", wonach dieses Referendum nicht erforderlich war, wenn die Bürgerschaft einstimmig beschloß. Die Praxis machte daraus etwas ganz anderes. In einem wahren ,, horror populi: auf keinen Fall Volksentscheid!“ (S. 419) änderte man die Verfassung eben nur, wenn Einstimmigkeit erreichbar war $^{37}$ was in den ersten 47 Jahren nur sechsmal der Fall war. Bei der Partialrevision von 1994 führte man dann die Möglichkeit der Verfassungsänderung mit parlamentarischer Zweidrittel-Mehrheit ein - und es war, als ob man die Schleusen geöffnet hätte. Schefold zählt neun Verfassungsänderungen seitdem, und inzwischen sind bis Juli 2012 weitere acht hinzugekommen ${ }^{38}$. Als „Verfassungsrevisionssucht“ geißelt Schefold dies mit Recht (S. 420). Beispiel Regierungssystem: Die neuere Verfassungsdogmatik und Verfassungsrechtsprechung entnehme dem Grundgesetz „das Konzept eines einheitlichen, über Parlament und Regierung vermittelten Legitimationszusammenhangs“ (S. 425), hinter dem der Verfassungsgeschichtler den „Schatten der einheitlichen Staatsgewalt“ erkennt, die sich nach der Wiener Schlußakte von 1820 im monarchischen Souverän verkörperte (S. 426). „In Teilen der neueren Literatur“ werde nun eine Angleichung Bremens an dieses „hierarchische Demokratieprinzip des einheitlichen Legitimationszusammenhangs“ postuliert, obwohl dies „dem Grundkonzept des Verfassungsstaats in Bremen“ widerspreche (S. 426): dem „Dualismus von Senat und Bürgerschaft“ (S. 427) in einem Kooperationsverhältnis. Gegen solche Modernisierungsbestrebungen ohne ein Bewußtsein ,der verfassungskulturellen Identität des Landes“ (S. 426) hält Schefold fest: „Es gilt, zunächst die Funktionsweise des tradierten bremischen Verfassungssystems zu verstehen. Man kann und soll-

37 Von einem ,unmöglichen Ergebnis“ sprach Schefold schon in dem ersten Beitrag dieses Kapitels (S. 401).

38 Am 12. Juli 2012 beschloß die Bürgerschaft vier weitere Verfassungsänderungen in Erster Lesung; vgl. jetzt das Änderungsgesetz vom 17.7.2012 (Brem. GBl. 2012 S. 354). 
te dann darin die Anregungen finden, auf neu entstandene Probleme zu reagieren und mit Instrumenten im Rahmen des Bremer Verfassungsmodells dieses fortzuentwickeln“ (S. 429).

Eine glänzende verfassungsgeschichtliche Analyse und noble Würdigung des Verfassungswerks von 1849.

\section{Zur Kommunalen Selbstverwaltung}

Dieses letzte Kapitel beginnt mit dem Beitrag ,Selbstverwaltungstheorien: Rudolf Gneist und Hugo Preuß", zuerst 2003 erschienen. Einleitend wendet sich Schefold gegen allzu einfache Entgegensetzungen dieser beiden Wissenschaftler; man solle sie eher „,in einer Entwicklungslinie sehen“ (S. 431). Entsprechend dem Abstand von anderthalb Generationen zwischen Gneist und Preuß skizziert Schefold zunächst die Phasen der Selbstverwaltung im 19. Jahrhundert. Eine Schlüsselstellung nimmt dabei der Freiherr v. Stein mit seiner preußischen Städteordnung von 1808 ein, für den diese Kommunalreform „nur ein erster Schritt“ einer umfassenden Staatsreform war, die über die Provinzen schließlich bis zu einer preußischen Nationalrepräsentation führen sollte. Da diese nie verwirklicht wurde, blieb jene „Stückwerk“, und ,dieser Widerspruch bestimmte in der Folge den Stellenwert der Selbstverwaltung“" (S. 434).

Dann kommen die Protagonisten ins Blickfeld. Gneists, des älteren, Ausgangspunkt war ein staatsrechtlicher; staatliche und kommunale Aufgaben unterschieden sich für ihn nicht qualitativ, sein Ziel war eine „obrigkeitliche Selbstverwaltung" (S. 440), worunter er eine mittelbare Staatsverwaltung, unterstützt durch ehrenamtliche örtliche Vertreter, verstand. Einen eigenen Wirkungskreis der Gemeinde lehnte er ab; das wären für ihn ,ebenso partikulare Sonderinteressen wie die der Junker“ (S. 439). Bei grundsätzlicher Anerkennung des Prinzips der Demokratie - auch er war ja von den Ideen von 1848 geprägt -, war Gneist doch von modernen demokratischen Legitimationsanforderungen weit entfernt, wie seine spätere Rechtfertigung des preußischen Drei-Klassen-Wahlrechts zeigt. Auch die Verwaltungsgerichtsbarkeit, zusammen mit dem „Rechtsstaat“ der „Kern von Gneists Selbstverwaltungslehre“, sollte mehr „die objektive Rechtmäßigkeit des Verwaltungshandelns sichern“ (S. 442), als subjektive Rechte der Bürger schützen.

Preuß, der jüngere, vertrat ebenfalls den einheitlichen Charakter der öffentlichen Aufgaben, übernahm aber das Steinsche Konzept der Eigenverantwortlichkeit der Gemeinden. Bei der Mitwirkung der Bürger befürwortete er entschieden die moderne demokratische, letztlich parlamentarische Legitimation. Brisant war Preuß' Vorstellung von Gemeinde, Staat und Reich als - grundsätzlich gleichwertige - Gebietskörperschaften. Wenn dann noch die kommunale Selbstverwal- 
tung als vorbildlich verstanden wurde, ja die Gemeinde als Modell, wurde „die Rechtfertigung des monarchischen Prinzips auf den höheren Ebenen brüchig" (S. 436).

Die theoretischen Positionen zweier solcher Wissenschaftler und deren Veränderungen darf man - ganz im Sinne des Schefold-Worts von der „Entwicklungslinie" - nicht hart oder gar konfrontativ darstellen. Wie Gneist das Selbstverwaltungsverständnis v. Steins weiterentwickelte, aber auch abwandelte (vgl. S. 439), so war etwa Preuß` Amtsverständnis dem Gneists (und v. Steins) durchaus „verwandt“ (S. 453); freilich ging er dann ,über die von Gneist idealisierte Ehrenamtlichkeit hinaus und betont(e) die von Gneist verkannte Notwendigkeit einer Professionalisierung der Gemeindeverwaltung" (S. 445). Und mit seiner Entscheidung für die parlamentarische Demokratie kann Preuß endlich „als Überwinder der von Gneist so leidenschaftlich verfochtenen obrigkeitsstaatlichen Grundlage der Selbstverwaltung betrachtet werden“ (S. 453) - obschon dessen wissenschaftliche Gesamtleistung „offenbar auch Preuß zum Bewunderer Gneists gemacht“ hat (S. 454). Selten formuliert Schefold denn auch hart: Bei der Frage, ob wegen der eigenständigen Legitimation der kommunalen Ebene auch ihre Aufgabenerfüllung als selbständig zu konzipieren sei, bezeichnet er den Gegensatz Preuß‘ zu Gneist als „diametral““ (S. 446).

Die größten Unterschiede zeigt Schefold abschließend lebensgeschichtlich. Gewiß hatte Gneist auch Zurücksetzungen erfahren, und im preußischen Verfassungskonflikt stand er auf der Seite der Opposition. Aber im weiteren Verlauf sollte er sich, wie Bismarck gesagt haben soll, derart „loyal und durch und durch gouvernemental“ (S. 441) entwickeln, daß er ,im Kaiserreich auf der Ebene des Reichs und Preußens maßgeblich zu wirken“ vermochte und endlich „zu den wichtigsten Wegbereitern der Entwicklung des deutschen öffentlichen Rechts in der zweiten Hälfte des 19. Jahrhunderts gehörte“ (S. 454). Preuß dagegen war schon in der Wissenschaft ein „Außenseiter“ (S. 454), was durch seine „linksfreisinnige politische Betätigung" und antisemitische Ressentiments noch verstärkt wurde (S. 455). In der Novemberrevolution 1918 wurde ihm dann das zuteil, ,wovon ein Staatsrechtslehrer nur träumen kann, die Vorbereitung einer völlig neuen Verfassung unter Realisierung eigener Vorstellungen“ (S. 454). Allerdings gilt dies nur mit herben Abstrichen: Preuß hat sich mit seinen Vorstellungen „Zwar teilweise, aber keinesfalls umfassend durchgesetzt“ (S. 455) - sein zentrales Bestreben der Neugliederung etwa scheiterte am Beharrungsvermögen der Einzelstaaten. Vor allem erstreckte sich bald die politische Ablehnung der Weimarer Reichsverfassung auf ihren Schöpfer, der seine letzten Jahre ,in erster Linie der Verteidigung jenes Verfassungswerks“ widmete (S. 455), eine tragische Situation, auch wenn dem 1925 Gestorbenen das Schlimmste acht Jahre später erspart blieb. 
In dem Beitrag „, Privatisierung in der Kommunalverwaltung “, 2004 an entlegener Stelle erschienen ${ }^{39}$, stellt Schefold zunächst im Überblick Aufstieg und Niedergang der Kommunalwirtschaft dar. Ursprünglich, zu v. Steins Zeiten, war die Kommunalisierung von Aufgaben als Entstaatlichung gedacht. Später weitete sich als Folge von Urbanisierung, Kriegs- und Nachkriegszeiten die kommunale Daseinsvorsorge immer mehr aus, bis dann die Idee der formellen Privatisierung aufkam, um die kommunalen Unternehmen betriebswirtschaftlich flexibler zu machen. Freilich folgten diese nun als Aktiengesellschaft oder Gesellschaft mit beschränkter Haftung organisierten Unternehmen alsbald einer gesellschaftsrechtlichen Eigenlogik - es ging nun um die Ertragslage, um die optimale Nutzung des eingesetzten Kapitals, um die Expansion des Geschäfts -, dem gegenüber ,die gesetzlich vorgesehene Steuerung durch das Trägergemeinwesen als Fremdkörper" erschien. Tendenziell unterschied sich das Verhalten eines solchen Unternehmens in öffentlicher Trägerschaft ,,von dem eines privaten Unternehmens des gleichen Geschäftszweigs nur wenig“" (S. 460), weswegen es auch als Konkurrenz wahrgenommen und dieser Wettbewerb als unlauter empfunden wurde - mit allen gemeinschafts- bzw. vergaberechtlichen Konsequenzen. Wird daraufhin das Ziel der „Wettbewerbsgleichheit“ öffentlicher und privater Unternehmen gesteckt, ja deren „Gleichstellung“ angestrebt, entfällt letztlich „,die Rechtfertigung öffentlicher Unternehmen“ (S. 460). Man könnte dann den Gemeinden nur raten, die noch existierenden kommunalen Unternehmen auch materiell zu privatisieren und sich damit aus der einschlägigen Aufgabenerfüllung zurückzuziehen.

Alsdann versucht Schefold die kommunal(verfassungs-)rechtlichen Weichen ausfindig zu machen, die bei diesem Weg in Richtung Selbstaufhebung falsch gestellt wurden. So erinnert er daran, daß ,jedenfalls die kommunalwirtschaftliche Betätigung zur Bewirkung von Leistungen, die einem öffentlichen Zweck dienen, von der Gewährleistung der kommunalen Selbstverwaltung erfaßt" ist (Art. 28 Abs. 2 Satz 1 GG; S. 461). Diese Kompetenz der Gemeinde begrenze „die grundrechtliche Position privater Konkurrenten“; das private Wettbewerbsrecht sei deshalb unanwendbar, mit ihm zu operieren „widersinnig“ (S. 462). Umgekehrt gebe es kommunalrechtliche Schranken gegen den ökonomischen Privatisierungsdruck, der auf den Kommunen laste. Eigenwirtschaftlichkeit von Einrichtungen dürfe nicht so strikt verlangt werden, daß sich deren Zweck wandle. Sonst könnte sich der Konflikt zuspitzen: „Entweder der öffentliche Zweck wird verfolgt, aber auch zu Lasten der Ertragskraft, dann ist das Unternehmen zulässig, aber regelmäßig nicht eigenwirtschaftlich. Oder der Eigenwirtschaftschreiben 36 (2004), S. 89-93 (Nr. 2). 
lichkeit wird der Vorrang gegeben, dann fehlt es am rechtfertigenden öffentlichen Zweck, und das Unternehmen ist unzulässig." (S. 463).

Als Ausweg, um diesen Zielkonflikt abzumildern, verweist Schefold auf die Unterscheidung zwischen Gewährleistungsverantwortung, die bei der öffentlichen Hand bleiben soll, und Erfüllungsverantwortung, also der Leistungserbringung, die privatisiert werden kann. Beispiele auf Bundesebene sind die Privatisierung von Post, Telekommunikation und Eisenbahnverkehr, jeweils verbunden mit einem grundgesetzlichen Infrastrukturauftrag. Dies sieht er als ein mögliches Vorbild für die kommunalen Unternehmen an, betont allerdings, daß ein Gelingen ,von Definition, faktischer Durchsetzung und vor allem Finanzierbarkeit des Infrastrukturauftrags“ abhänge (S. 463). Letztlich fürchtet er, daß die Gewährleistung eines solchen Infrastrukturauftrags die Kommunen überfordern könnte.

In dem Beitrag ,Zur Gestalt der Regionen“, zuerst erschienen 2005 in der Festschrift für Manfred Zuleeg, widmet sich Schefold einigen Problemen, die für die Gestaltung einer föderativen Ordnung in Europa bestehen. Als erstes stellt er die Regionen im geltenden Gemeinschaftsrecht vor. Die EU hat sie auf zwei Wegen anerkannt: Durch die Einbeziehung dieser unteren Ebenen in das Ziel einer harmonischen, ausgewogenen und nachhaltigen Entwicklung des Wirtschaftslebens, zu dem der Europäische Regionalfonds beitragen soll. Ferner schuf man den Ausschuß der Regionen, wenn auch nur als beratendes Gremium. Eine Schwachstelle dieser Regelungen sieht Schefold darin, daß der Begriff der Region unscharf sei und mannigfache Formen umfasse. Besonderes Augenmerk verdienten jedenfalls die grenzüberschreitenden Regionen (vgl. Art. 24 Abs. 1a GG: ,grenznachbarschaftliche Einrichtungen“), für Schefold naheliegend die Regio Basiliensis. Darin sieht er eine „Paradigmen-Erweiterung“, daß die Mitgliedstaaten der Union nicht mehr ,als impermeable Einheiten“ angehören (S. 467). Im Übrigen sei bei aller Unbestimmtheit jedenfalls der Trend in „Richtung auf eine innere Ausdifferenzierung der Mitgliedstaaten“ unverkennbar (S. 468). Den regionalen Gebietskörperschaften garantiert die Union immerhin, wie Schefold dartut, „Existenz, Legitimation und Aufgabenbereich“ (S. 472) ${ }^{40}$.

Neben diese gemeinschaftsrechtliche Anerkennung stellt Schefold den Beitrag des Europarats, der für ihn besonders wichtig ist, weil er in der Sachverständigengruppe des Kongresses der Gemeinden und Regionen Europas (KGRE) als von Deutschland bestellter Experte gewirkt hat (vgl. S. 475 Fn. 47; ferner das Vorwort der Herausgeber, S. 7). Dort hatte man zunächst versucht, die Erfolgs-

40 Schefold ging bei der Abfassung seines Beitrags noch von dem damals vorliegenden Entwurf einer Europäischen Verfassung aus. Die einschlägigen Vorschriften sind durch den Vertrag von Lissabon in die Konsolidierte Fassung des EUV übernommen worden, z.B. Art. I-5 VVE Abs. 1 Satz 1 in Art. 4 Abs. 2 Satz 1 EUV. 
geschichte der „Europäischen Charta der kommunalen Selbstverwaltung“ zu wiederholen, die 1985 unterzeichnet wurde und 1988 in Kraft trat. Aber das seit 1997 verfolgte Projekt einer „Europäischen Charta der regionalen Selbstverwaltung“ ließ sich viel schwerer an. Während einige Regionalisierungsmodelle gelungen erscheinen - z.B. in Spanien als Antwort auch auf sprachlich-ethnische Differenzen (vgl. S. 480) -, stößt der ganze Ansatz auf grundsätzliche Skepsis in anderen Staaten, die Regionalisierung mit Sezessionismus, Bedrohung der nationalen Integrität oder zumindest mit Machtverlust der Zentralregierung verbinden. Der Versuch, diesen Befürchtungen gerecht zu werden, führte, wie Schefold darstellt, zu hoher Komplexität des Entwurfs, zugleich bei der Verbindlichkeit zu einem ,système à la carte“ (S. 481) und damit zu Intransparenz und letztlich zu Zweifeln an der Möglichkeit überhaupt, hier staatsvertragliche Bindungen zu erreichen.

Als Perspektive sieht Schefold, wenn die Schwierigkeiten sich als unüberwindbar erweisen sollten, die Entwicklung eines Modells der Regionalisierung an, ,das die geltenden Ansätze zusammenführt, systematisch darstellt und daraus Folgerungen ableitet" (S. 482). So könnten die Europäische Union und der Europarat bzw. ihre einschlägigen Unterorganisationen einen „Code européen“ für die regionale Autonomie als „Orientierungshilfe“ entwickeln (S. 483), aus dem später einmal eine verbindliche Regelung entstehen könnte.

Mit diesem realistisch-skeptischen Ende hat Schefold gewiß richtig gesehen. Nachdem ein zweiter Entwurf einer „Europäischen Charta für regionale Demokratie“ (2008) vom Ministerrat nicht akzeptiert wurde, formulierte dieser 2009 selbst einen „Bezugsrahmen für regionale Demokratie“, der einerseits das Scheitern des Plans einer rechtsverbindlichen Konvention bedeutete, andererseits sich eines Tages eben doch als Kern einer bindenden Konvention der regionalen Selbstverwaltung erweisen könnte ${ }^{41}$.

Der letzte Beitrag dieses Kapitels - zugleich der Abschluß des ganzen Bandes -, überschrieben ,Der Schutz der kommunalen Selbstverwaltung durch den Europarat “, zuerst erschienen 2007 im Liber amicorum Luzius Wildhaber, untersucht den Rechtsschutz der kommunalen Selbstverwaltung auf europäischer Ebene, ist also vom Arbeitsgebiet her dem vorigen Beitrag eng verwandt und profitiert wie jener von dem Expertenwirken Schefolds bei dem KGRE.

Die Differenzierung, die bereits das nationale Recht kennt, etwa in Deutschland zwischen der Individualverfassungsbeschwerde und der besonderen Kommunalverfassungsbeschwerde, setzt sich im Europarecht fort. Ein Rechtsschutz, rahmen für regionale Demokratie“, in: Europäisches Journal für Minderheitenfragen 3 (2010), S. 114-128. 
wie ihn die Europäische Menschenrechtskonvention gewährt, ist z.B. in der Europäischen Sozialcharta nicht vorgesehen und erst recht nicht für die kommunale Selbstverwaltung. Das von der KGRE praktizierte Monitoring-System bleibt bei aller Tendenz ,zur Objektivität und zur Annäherung an ein gerichtsähnliches Verfahren“ (S. 496): Eingehen auf konkrete Streitfragen, Abstandswahrung, indem Mitglieder aus anderen Signatarstaaten als dem betroffenen Bericht erstatten, Sorge für juristische Korrektheit durch die Begutachtung unabhängiger Experten - politische Gestaltung durch Berichte, Entschließungen und Empfehlungen, aber nicht richterliche Rechtsanwendung.

Eine Änderung ist hier eingetreten, als der KGRE begann, eigentliche Beschwerden wegen Verletzung der Charta der kommunalen Selbstverwaltung entgegenzunehmen, vor allem, seitdem die Sachverständigengruppe 2005 für deren Behandlung Verfahrensregeln aufgestellt hat. Wenn als Ergebnis dieses „formalisierte[n] Beschwerdeverfahren[s]“ (S. 498) nicht eine politische Lösung empfohlen, sondern ,in Anwendung der Charta eine bestimmte Lösung als geboten erklärt und damit ein konkreter Rechtsstandpunkt beschlossen wird“ (S. 496), kommt das einer europarechtlichen Kommunalverfassungsbeschwerde durchaus nahe. So begrüßenswert diese Entwicklung aus politischen Gründen auch erscheinen mag - Schefold, selbst involviert, spricht als Rechtswissenschaftler untadelig korrekt von ,einer Praxis, die die Satzung des Europarats überaus expansiv interpretiert und weitergebildet hat" (S. 498).

Freilich bleibt die Bindungswirkung ein Problem, zu dem sich Schefold dreifach äußert. Nüchtern: „Auch nicht verbindliche und keine konkreten Rechte begründenden völkerrechtlichen Äußerungen sind deshalb nicht bedeutungslos, sondern können die Wirkungen von soft law haben und die Praxis, namentlich die Auslegung und Anwendung des einzelstaatlichen Rechts, beeinflussen“ (S. 499). Ironisch: „Das Fehlen rechtlicher Verbindlichkeit schließt die Überzeugungskraft der Feststellungen nicht aus“ (S. 500). Und realistisch-skeptisch: „Von einer solchen Überzeugungswirkung zur Bindungswirkung ist ein weiter Weg“' (S. 500).

\section{Schluß}

Dimitrios Th. Tsatsos, der Gründer des Instituts für Europäische Verfassungswissenschaften an der Fernuniversität Hagen, hat diesen Band angeregt; dem Andenken dieses Freundes, der während der Vorbereitung verstarb, ist er gewidmet. Die Mitherausgeber zitieren Tsatsos, daß es ihm um ,die Höhepunkte der wissenschaftlichen Leistungen eines brillanten Denkers“ ging (S. 7) - ein Vorhaben, dessen Wunsch zur Ehrung des Autors völlig berechtigt ist, das aber kaum durch das Genre einer Aufsatzsammlung erreicht werden kann. Dafür kä- 
men eher die selbständigen Schriften Schefolds in Betracht, deren Reprint freilich verlegerisch noch schwieriger wäre, zumal man dann zu vierstelligen Seitenzahlen käme. Tatsächlich handelt es sich um eine Auswahl der besten unter den kleineren Arbeiten Schefolds, wobei zum Kriterium der Qualität noch andere ein- bzw. ausschließende Gesichtspunkte hinzukamen (nur schwer greifbar - allein sechs Festschriftenbeiträge -, nicht fremdsprachlich). Dies soll keine Beckmesserei sein, sondern sozusagen in Schefoldscher Nüchternheit zusammenfassen, was der löbliche Band enthält.

Nicht ganz gelungen erscheint der Titel. „Bewahrung der Demokratie“ klingt zu besorgt. Im Vorwort formulieren die Mitherausgeber schon kräftiger, der inhaltliche Schwerpunkt der Beiträge sei „die Durchsetzung und Erhaltung demokratischer Prozesse“ (S. 7). Und viele der hier versammelten Aufsätze handeln ganz positiv gestimmt von Entwicklung, Entfaltung und Gestaltung der Demokratie. 